\title{
Optimized Schwarz methods for a diffusion problem with discontinuous coefficient
}

\author{
Martin J. Gander • Olivier Dubois
}

Received: 11 March 2014 / Accepted: 11 June 2014 / Published online: 25 July 2014

(C) Springer Science+Business Media New York 2014

\begin{abstract}
We study non-overlapping Schwarz methods for solving a steady-state diffusion problem in heterogeneous media. Various optimized transmission conditions are determined by solving the corresponding min-max problems; we consider different choices of Robin conditions and second order conditions. To compare the resulting methods, we analyze the convergence in two separate asymptotic regimes: when the mesh size is small, and when the jump in the coefficient is large. It is shown that optimized two-sided Robin transmission conditions are very effective in both regimes; in particular they give mesh independent convergence. Numerical experiments are presented to illustrate and confirm the theoretical results.
\end{abstract}

Keywords Optimized Schwarz methods - Optimized transmission conditions · Domain decomposition · Parallel preconditioning

\section{Introduction}

The simulation of flow in heterogeneous porous media is an important problem that arises in many engineering applications: oil recovery, earthquake prediction, underground disposal of nuclear waste, etc. In these applications, the computational

This work was supported in part by scholarships from NSERC, FQRNT and McGill University.

M. J. Gander (殴

Section de mathématiques, Université de Genève,

2-4 rue du Lièvre, C.P. 64 1211, Genève 4, Suisse

e-mail: Martin.Gander@math.unige.ch

O. Dubois

Department of Mathematics, John Abbott College, 21275

Lakeshore Road, Sainte-Anne-de-Bellevue, H9X 3L9, Québec, Canada

e-mail: olivier.dubois@johnabbott.qc.ca 
domain often consists of several regions with significantly different physical properties. In the mathematical model, this translates into a partial differential equation with discontinuous coefficients.

For large numerical computations with those problems, domain decomposition is a natural idea: a non-overlapping decomposition is directly suggested by the different physical regions. Classical Schwarz methods require overlap to converge (see for example the books [33], [35]) and thus cannot be used in this context. Modern nonoverlapping domain decomposition methods such as FETI and its variants (see [9], [35] and references therein) were developed and analyzed; they give mesh independent convergence. These methods are based on either Dirichlet or Neumann coupling conditions between subdomains.

More general transmission conditions were then proposed for the Schwarz method in order to obtain a converging method without overlap [26], or to accelerate the convergence $[20,32,34]$. This led in particular to optimized Schwarz methods, in which transmission conditions are optimized for performance. This approach was first introduced by Japhet [22] for advection-diffusion problems, with further developments appearing in [25], [23] and [24]. Later, optimized Schwarz methods were thoroughly analyzed for symmetric positive definite problems [11] and for the Helmholtz equation [10], [16]. The idea of optimized transmission conditions is also employed to improve the performance of Schwarz Waveform Relaxation methods for parabolic problems $[1,13,31]$, and hyperbolic problems $[12,15]$. In all these studies, the model problem considered has constant coefficients over the entire computational domain, and the results can be applied in general for smoothly varying coefficients .

For problems in heterogeneous media, some domain decomposition methods with Robin transmission conditions have appeared in the litterature. In [19], a Robin-Robin preconditioner is proposed for advection-diffusion problems having discontinuous viscosity coefficients, and an estimate for the convergence factor is derived for a model problem. In [7] and [3], optimized Robin conditions are mentioned but the optimization problem is not fully solved; instead, an approximate choice is made for the Robin parameters. In [17] and [18], optimized Schwarz methods are designed at the algebraic level, where the problem is discretized in the tangential direction to the interface. The proposed parameters are the same along the entire interface. Some work has also been developed on optimized Schwarz waveform relaxation for parabolic problems with discontinuous coefficients, for example in [14] and [2]. More recently, Maday and Magoulès have studied optimized Schwarz methods for a diffusion problem with discontinuous coefficient in [27], [28] and [29]. They derived optimized parameters, but under certain assumptions only, and without always proving that these parameters are the (unique) solution of the min-max problem.

In this paper, we consider the same model problem as Maday and Magoulès, i.e. a simple diffusion problem with discontinuous coefficient, and derive optimized Schwarz methods by thoroughly and rigorously solving the associated min-max problems. These optimization problems share similarities with the Chebyshev best approximation problem, and indeed the minimizers are often characterized by an equioscillation property. Some general results in this direction appear in [1] in the 
context of waveform relaxation. However, for second order elliptic problems, the equioscillation property does not always yield the best transmission conditions: a counterexample can be found in [4] for the advection-diffusion equation, and another one appears in this paper. Therefore, for each choice of transmission conditions, we analyze carefully the min-max problem in order to obtain precise formulas or characterizations for the parameters that always give the best performance for the Schwarz method. The analysis is restricted to the case of two subdomains only, because optimized conditions are used to improve the local coupling between neighboring subdomains. When many subdomains are used, a coarse space correction is necessary in order for the convergence to be independent of the number of subdomains [6]; we consider this a separate issue that we do not investigate here.

This paper is organized as follows. In Section 2, we introduce the model problem and the Schwarz iteration, perform a Fourier analysis of the convergence, and discuss optimal coupling conditions. In Section 3, we review the basic ideas behind optimized Schwarz methods, and then proceed to analyze several choices of optimized transmission conditions for our model problem. The asymptotic convergence factor is studied for the case of small mesh sizes and strong heterogeneity. For comparison purposes, Section 4 contains a short discussion of the Dirichlet-Neumann method. In Section 5, we show that all our results can be directly extended to problems with anisotropic diffusion tensors. Finally, in Section 6, numerical experiments are presented to illustrate the convergence analysis.

\section{A diffusion model problem}

We look at the case of a steady-state diffusion problem

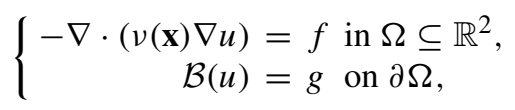

where the scalar diffusion coefficient $\nu(\mathbf{x})$ is the piecewise constant function

$$
v(\mathbf{x})= \begin{cases}v_{1} & \text { for } \mathbf{x} \in \Omega_{1}, \\ v_{2} & \text { for } \mathbf{x} \in \Omega_{2} .\end{cases}
$$

Here, $\Omega_{1}$ and $\Omega_{2}$ form a natural decomposition of the domain $\Omega$ into nonoverlapping subdomains, and we denote by $\Gamma$ the interface between the subdomains. Using physical coupling conditions between the subdomains, the problem can also be written equivalently (in a weak sense) in a multidomain formulation

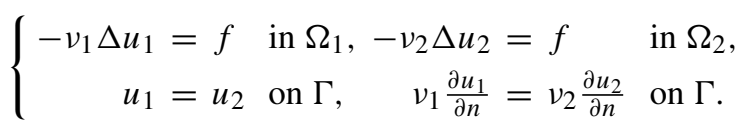

The matching conditions impose the continuity of the solution and of its flux across the interface $\Gamma$.

As usual for the derivation of optimized Schwarz methods, we consider a model problem on the infinite plane $\Omega=\mathbb{R}^{2}$, with the subdomains

$$
\Omega_{1}=(-\infty, 0) \times \mathbb{R}, \quad \Omega_{2}=(0, \infty) \times \mathbb{R} .
$$


To be consistent with the physical coupling conditions in (1), we use a parallel Schwarz iteration with general transmission conditions of the form

$$
\begin{aligned}
& \left\{\begin{array}{rlrl}
-v_{1} \Delta u_{1}^{n} & =f & & \text { in } \Omega_{1}, \\
\left(v_{1} \partial_{x}+\mathcal{S}_{1}\right) u_{1}^{n}(0, y) & =\left(v_{2} \partial_{x}+\mathcal{S}_{1}\right) u_{2}^{n-1}(0, y) & \text { for } y \in \mathbb{R},
\end{array}\right. \\
& \left\{\begin{array}{rlrl}
-v_{2} \Delta u_{2}^{n} & =f & & \text { in } \Omega_{2}, \\
\left(v_{2} \partial_{x}-\mathcal{S}_{2}\right) u_{2}^{n}(0, y) & =\left(v_{1} \partial_{x}-\mathcal{S}_{2}\right) u_{1}^{n-1}(0, y) & \text { for } y \in \mathbb{R},
\end{array}\right.
\end{aligned}
$$

where $\mathcal{S}_{j}$ are linear operators acting in the $y$ direction only. By linearity, it will be sufficient to consider only the homogeneous case, $f \equiv 0$, in the convergence analysis.

\subsection{Fourier analysis}

Our simple model problem allows us to use a Fourier transform in the $y$ variable,

$$
\mathcal{F}_{y}(u(x, y))=\hat{u}(x, k):=\int_{-\infty}^{\infty} u(x, y) e^{-i y k} d y,
$$

to analyze the convergence of the Schwarz method (2). Suppose the operators $\mathcal{S}_{j}$ have Fourier symbols $\sigma_{j}(k)$. In Fourier space, the partial differential equation in $\Omega_{j}$ becomes a second order ODE in $x$ (for each fixed $k$ ),

$$
\begin{aligned}
& \left\{\begin{aligned}
-v_{1}\left(\partial_{x x}-k^{2}\right) \hat{u}_{1}^{n} & =0 & & \text { for } x<0, k \in \mathbb{R}, \\
\left(v_{1} \partial_{x}+\sigma_{1}(k)\right) \hat{u}_{1}^{n}(0, k) & =\left(v_{2} \partial_{x}+\sigma_{1}(k)\right) \hat{u}_{2}^{n-1}(0, k) & & \text { for } k \in \mathbb{R},
\end{aligned}\right. \\
& \left\{\begin{aligned}
-v_{2}\left(\partial_{x x}-k^{2}\right) \hat{u}_{2}^{n} & =0 & & \text { for } x>0, k \in \mathbb{R}, \\
\left(v_{2} \partial_{x}-\sigma_{2}(k)\right) \hat{u}_{2}^{n}(0, k) & =\left(v_{1} \partial_{x}-\sigma_{2}(k)\right) \hat{u}_{1}^{n-1}(0, k) & & \text { for } k \in \mathbb{R},
\end{aligned}\right.
\end{aligned}
$$

with characteristic roots $\lambda_{ \pm}(k):= \pm|k|$. By requiring that the solutions in each subdomain be bounded at infinity, we find solutions of the form

$$
\hat{u}_{1}^{n}(x, k)=A^{n}(k) e^{|k| x}, \quad \hat{u}_{2}^{n}(x, k)=B^{n}(k) e^{-|k| x} .
$$

Applying the transmission conditions coupling the two subdomains, we obtain the convergence factor

$$
\rho\left(k, \sigma_{1}, \sigma_{2}\right):=\left|\frac{\hat{u}_{j}^{n+1}(0, k)}{\hat{u}_{j}^{n-1}(0, k)}\right|=\left|\frac{\sigma_{1}(k)-v_{2}|k|}{\sigma_{1}(k)+v_{1}|k|} \cdot \frac{\sigma_{2}(k)-v_{1}|k|}{\sigma_{2}(k)+v_{2}|k|}\right| .
$$

Note that at $k=0$, the convergence factor equals 1 , for any choice of $\sigma_{j}$, i.e. the frequency $k=0$ can never converge. Of course, this makes sense because the solution to the global model problem on $\mathbb{R}^{2}$ is only unique up to a constant. This will not cause any difficulty: we will only consider strictly positive frequency components, $|k| \geq k_{1}>0$, because in practice the computational domain will be bounded with appropriate boundary conditions and the problem will be well-posed. 


\subsection{Optimal operators}

The operators $\mathcal{S}_{j}$ (or equivalently the symbols $\sigma_{j}$ ) are still free to be chosen at this point. It is straightforward, by inspection of (3), to see that we get optimal convergence, in two iterations, if we choose the symbols

$$
\sigma_{1}^{o p t}(k)=v_{2}|k|, \quad \sigma_{2}^{o p t}(k)=v_{1}|k| .
$$

These symbols are not polynomials in $k$, and thus the corresponding operators in real space,

$$
\mathcal{S}_{j}^{o p t}(u(0, y))=\mathcal{F}_{y}^{-1}\left(\sigma_{j}^{o p t}(k) \hat{u}(0, k)\right),
$$

are nonlocal in $y$. In fact, the operators $\mathcal{S}_{j}^{o p t}$ are Dirichlet-to-Neumann maps, which involve solving a problem in the adjacent subdomain. We can define these operators in general as follows: consider a bounded domain $\Omega$ with a nonoverlapping decomposition $\left\{\Omega_{1}, \Omega_{2}\right\}$, let $\Gamma$ denote the interface between the subdomains and let $\frac{\partial}{\partial n}$ denote the normal derivative across $\Gamma$ in the outward direction with respect to $\Omega_{1}$. We can define the operator $\mathcal{S}_{1}^{\text {opt }}: H_{00}^{1 / 2}(\Gamma) \rightarrow H^{-1 / 2}(\Gamma)$ via $\mathcal{S}_{1}^{\text {opt }}(u):=-v_{2} \frac{\partial w}{\partial n}$ where $w$ solves the problem

$$
-v_{2} \Delta w=f \quad \text { in } \Omega_{2}, \quad w=u \quad \text { on } \Gamma .
$$

Similarly, we can define the operator $\mathcal{S}_{2}^{\text {opt }}: H_{00}^{1 / 2}(\Gamma) \rightarrow H^{-1 / 2}(\Gamma)$ via $\mathcal{S}_{2}^{o p t}(u):=v_{1} \frac{\partial v}{\partial n}$ where $v$ solves the problem

$$
-v_{1} \Delta v=f \quad \text { in } \Omega_{1}, \quad v=u \quad \text { on } \Gamma .
$$

It is easy to verify that, using these nonlocal operators, the Schwarz iteration converges in two iterations, for any initial guesses $u_{j}^{0}, j=1,2$. These Dirichlet-toNeumann operators can be generalized to the case of a domain decomposition into $M$ strips, and yield an optimal convergence in $M$ iterations, see [32].

The optimal operators are easy to characterize in one dimension. Consider a bounded interval $\Omega$, with homogeneous Dirichlet boundary conditions on the boundary, and a general diffusion coefficient $v(x)$. Consider a partition of $\Omega$ into two nonoverlapping subintervals $\Omega_{1}$ and $\Omega_{2}$. In this case, the optimal transmission conditions are simple Robin conditions

$$
\mathcal{S}_{1}^{o p t}(u)=\left[\int_{\Omega_{2}} v(x)^{-1} d x\right]^{-1} u, \quad \mathcal{S}_{2}^{o p t}(u)=\left[\int_{\Omega_{1}} v(x)^{-1} d x\right]^{-1} u,
$$

as was pointed out in [26].

\section{Optimized Schwarz methods}

Being nonlocal, the optimal operators are not convenient for implementation; they are computationally costly. Instead, we would like to find good local transmission conditions that still give very fast convergence of the Schwarz iteration (2). The idea is to fix a class $\mathcal{C}$ of convenient transmission conditions to consider, and uniformly 
optimize the convergence factor over a range of relevant frequencies for our problem. This leads to a min-max problem of the form

$$
\min _{\sigma_{j} \in \mathcal{C}}\left(\max _{k_{1} \leq k \leq k_{2}} \rho\left(k, \sigma_{1}, \sigma_{2}\right)\right) .
$$

Although the convergence factor $\rho$ was computed for a continuous model problem on the infinite plane, we impose bounds on the frequency range by incorporating information about the actual problem we intend to solve. For example, if we wish to compute a solution over a bounded domain with homogeneous Dirichlet boundary conditions, then the minimal frequency component of the solution can be estimated by $k_{1}=\frac{\pi}{H}$ where $H$ is the diameter of the subdomain. The lower bound $k_{1}$ could also be taken to be the smallest frequency not resolved by the coarse grid, if one is used, see for example [6]. Moreover, if the solution is computed numerically on a grid with grid spacing $h$ on the interface, then the maximum frequency which can be represented on this grid is typically estimated by $k_{2}=\frac{\pi}{h}$.

Before analyzing several classes $\mathcal{C}$ of transmission conditions and solving the associated min-max problem, let us state sufficient conditions on $\sigma_{1}$ and $\sigma_{2}$ that guarantee convergence of the Schwarz iteration.

\section{Theorem 1 Under the condition}

$$
0<\sigma_{2}(k) \leq \sigma_{1}(k) \quad \text { if } v_{1}<v_{2}, \quad 0<\sigma_{1}(k) \leq \sigma_{2}(k) \quad \text { if } v_{2}<v_{1},
$$

for all $k \neq 0$, the Schwarz iteration (2) converges for all nonzero frequencies,

$$
\rho\left(k, \sigma_{1}, \sigma_{2}\right)<1, \quad \text { for } k \neq 0 .
$$

Proof For convergence, we want

$$
\left|\frac{\sigma_{1}-v_{2}|k|}{\sigma_{1}+v_{1}|k|} \cdot \frac{\sigma_{2}-v_{1}|k|}{\sigma_{2}+v_{2}|k|}\right|<1,
$$

which can be written as two separate inequalities

$$
-\left(\sigma_{1}+v_{1}|k|\right)\left(\sigma_{2}+v_{2}|k|\right)<\left(\sigma_{1}-v_{2}|k|\right)\left(\sigma_{2}-v_{1}|k|\right)<\left(\sigma_{1}+v_{1}|k|\right)\left(\sigma_{2}+v_{2}|k|\right) \text {. }
$$

By expanding the products, we find that the rightmost inequality holds if and only if $\sigma_{1}+\sigma_{2}>0$. For the leftmost inequality, we want

$$
0<\sigma_{1} \sigma_{2}+|k|\left(v_{1}-v_{2}\right)\left(\sigma_{2}-\sigma_{1}\right)+v_{1} v_{2} k^{2} .
$$

Simple sufficient conditions for this inequality to hold are $\sigma_{1} \sigma_{2} \geq 0$ and $\left(v_{1}-v_{2}\right)\left(\sigma_{2}-\sigma_{1}\right) \geq 0$ (these are clearly not necessary conditions). Combined with the previous conclusion that $\sigma_{1}+\sigma_{2}>0$, these reduce to the conditions (5).

In the following subsections, we consider four choices of transmission conditions and their corresponding min-max problems: three different types of Robin conditions (zeroth order approximations of $\sigma_{j}^{o p t}$ ), and one choice of second order conditions. In all cases, the conditions of Theorem 1 will turn out to be satisfied, thus guaranteeing 
convergence when using optimized conditions. Moreover, for a straight interface, when $\sigma_{j}(k)>0$ the subproblems are well-posed.

In the following, we will say that two min-max problems are equivalent if they have the same minimum value and they have the same set of global minimizers.

\subsection{Optimized Robin conditions: first version}

Let us first derive optimized Robin transmission conditions with only one free parameter. The most obvious way to reduce $\sigma_{1}$ and $\sigma_{2}$ to one degree of freedom is to choose

$$
\sigma_{1}(k)=\sigma_{2}(k)=p, \quad \text { with } p \in \mathbb{R} .
$$

We can already see that this may not be the best choice, by noticing that the optimal operators (4) are scaled differently in terms of $v_{1}$ and $v_{2}$, and appropriate conditions should probably imitate this scaling (see Section 3.2). Nonetheless, we still fully analyze this case for completeness and comparison purposes. A similar analysis of this choice of conditions can be found in [27, 29], but we offer here a more complete description of the optimized parameter, not only asymptotically, and show that it is not always obtained by equioscillation.

For this choice, we can write the convergence factor as

$$
\rho(k, p)=\left|\frac{\left(p-v_{-}|k|\right)\left(p-v_{+}|k|\right)}{\left(p+v_{-}|k|\right)\left(p+v_{+}|k|\right)}\right|,
$$

where we introduce the notation

$$
\nu_{-}:=\min \left\{v_{1}, v_{2}\right\}, \quad v_{+}:=\max \left\{v_{1}, v_{2}\right\} .
$$

We now wish to find the best value for the parameter $p$ in the following sense: it should minimize the convergence factor uniformly over a relevant range of frequencies. This is stated as the min-max problem

$$
\min _{p \in \mathbb{R}}\left(\max _{k_{1} \leq k \leq k_{2}} \rho(k, p)\right) .
$$

We look only at a positive range of frequencies, $k_{1}>0$, since the convergence factor is an even function of $k$. This means we can replace $|k|$ by $k$ for simplicity in the analysis. As a first step towards solving the optimization problem $\left(M_{1}\right)$, we show how to reduce the search range for $p$.

Lemma 1 (Restricting the range for $p$ ) The min-max problem $\left(M_{1}\right)$ is equivalent to the problem where we minimize over $p$ only in the interval $\left[v_{-} k_{1}, v_{+} k_{2}\right]$.

Proof First, we can restrict ourselves to the case $p>0$, by noticing that

$$
\rho(k, p)<\rho(k,-p), \quad \text { whenever } p>0, \forall k>0 .
$$

From Theorem 1, assuming that $p>0$ ensures convergence at this point. Suppose now that $p \notin\left[v_{-} k_{1}, v_{+} k_{2}\right]$. Then, we have

$$
\left(p-v_{-} k\right)\left(p-v_{+} k\right)>0 \text { for all } k \in\left[k_{1}, k_{2}\right],
$$


and so we can write the convergence factor without absolute values

$$
\rho(k, p)=\frac{\left(p-v_{-} k\right)\left(p-v_{+} k\right)}{\left(p+v_{-} k\right)\left(p+v_{+} k\right)} .
$$

Taking a derivative with respect to $p$, we find

$$
\frac{\partial \rho}{\partial p}=\frac{2 k\left(v_{-}+v_{+}\right)\left(p^{2}-v_{-} v_{+} k^{2}\right)}{\left(p+v_{-} k\right)^{2}\left(p+v_{+} k\right)^{2}} .
$$

In the case $p<v_{-} k_{1}$, we have

$$
p^{2}<v_{-}^{2} k_{1}^{2} \leq v_{-} v_{+} k^{2}, \quad \text { which implies that } \frac{\partial \rho}{\partial p}<0, \quad \forall k \in\left[k_{1}, k_{2}\right] .
$$

Thus, increasing $p$ will make the convergence factor decrease uniformly on $\left[k_{1}, k_{2}\right]$. Similarly, in the case $p>v_{+} k_{2}$, we have

$$
p^{2}>v_{+}^{2} k_{2}^{2} \geq v_{-} v_{+} k^{2}, \quad \text { which implies that } \frac{\partial \rho}{\partial p}>0, \quad \forall k \in\left[k_{1}, k_{2}\right] .
$$

Thus, decreasing $p$ will make the convergence factor decrease uniformly over $k$. This shows that any minimizer $p$ solving problem $\left(M_{1}\right)$ cannot be less than $v_{-} k_{1}$, nor can it be greater than $v_{+} k_{2}$.

We now turn to the behavior of $\rho(k, p)$ as a function of $k$.

Lemma 2 (Local maxima in $k$ ) Let $k_{c}(p):=\left(v_{-} v_{+}\right)^{-\frac{1}{2}} p$. For fixed $p$, we can write the maximum value of $\rho(k, p)$ as

$$
\max _{k_{1} \leq k \leq k_{2}} \rho(k, p)= \begin{cases}\max \left\{\rho\left(k_{1}, p\right), \rho\left(k_{c}(p), p\right), \rho\left(k_{2}, p\right)\right\} & \text { if } k_{c}(p) \in\left[k_{1}, k_{2}\right], \\ \max \left\{\rho\left(k_{1}, p\right), \rho\left(k_{2}, p\right)\right\} & \text { if } k_{c}(p) \notin\left[k_{1}, k_{2}\right] .\end{cases}
$$

Proof By differentiating $\rho(k, p)$ with respect to $k$, we find that

$$
\frac{\partial \rho}{\partial k}=0 \quad \text { if and only if } \quad k=k_{c}(p)=\frac{p}{\sqrt{\nu_{-} \nu_{+}}} .
$$

It is easy to check that this critical point $k_{c}$ is a local maximum of $\rho$. In fact, by looking at the sign of $\frac{\partial \rho}{\partial k}$ for a given $p>0$, we observe that, with respect to $k$,

$$
\rho(k, p) \text { is }\left\{\begin{array}{l}
\text { strictly decreasing for } k \in\left[0, \frac{p}{v_{+}}\right) \cup\left(\frac{p}{\sqrt{v_{-} v_{+}}}, \frac{p}{v_{-}}\right), \\
\text {strictly increasing for } k \in\left(\frac{p}{v_{+}}, \frac{p}{\sqrt{\nu_{-} v_{+}}}\right) \cup\left(\frac{p}{v_{-}}, \infty\right) .
\end{array}\right.
$$

By observing that the local maximum $k_{c}(p)$ does not always lie in the interval $\left[k_{1}, k_{2}\right]$ for some values of $p$, we can express the maximum magnitude of the convergence factor as stated in the lemma.

There are not enough degrees of freedom to always be able to match the value at the three possible local maxima (to obtain equioscillation). In fact, the value of the 
convergence factor at the interior local maximum $k_{c}(p)$ simplifies to

$$
\rho\left(k_{c}(p), p\right)=\frac{\left(\sqrt{\nu_{+}}-\sqrt{\nu_{-}}\right)^{2}}{\left(\sqrt{\nu_{+}}+\sqrt{\nu_{-}}\right)^{2}}=: R_{c},
$$

which is, surprisingly, independent of the parameter $p$. In other words, $R_{c}$ is a fixed value that cannot be improved by varying the parameter $p$.

The following theorem provides a complete characterization and formulas for the minimizers of the min-max problem $\left(M_{1}\right)$. Although this is the first min-max problem we study here, the result and its proof are the most complex of this paper. Depending on the case, there can be a unique minimizer, two distinct minimizers, or a full interval of minimizers. The result is stated in terms of the ratio of coefficients and the ratio of frequencies

$$
\mu:=\frac{v_{+}}{v_{-}}, \quad k_{r}:=\frac{k_{2}}{k_{1}} .
$$

Theorem 2 (Optimized Robin parameter: first version) Let

$$
f(\mu):=\left[(\mu+1)^{2}+(\mu-1) \sqrt{\mu^{2}+6 \mu+1}\right](4 \mu)^{-1} .
$$

(i) If $k_{r} \geq f(\mu)$, then one value of $p$ minimizing the convergence factor is $p^{*}=$ $\sqrt{\nu_{-} \nu_{+} k_{1} k_{2}}$. This minimizer $p^{*}$ is unique when $\rho\left(k_{1}, p^{*}\right) \geq R_{c}$. Otherwise, the minimum is also attained for any $p$ chosen in a closed interval around $p^{*}$.

(ii) If $k_{r}<f(\mu)$, then two distinct values of $p$ attain the minimum; they can be obtained by solving

$$
\rho\left(k_{1}, p^{*}\right)=\rho\left(k_{2}, p^{*}\right)
$$

in the intervals $\left[v_{-} k_{1}, \sqrt{\nu_{-} v_{+}} k_{1}\right]$ and $\left[\sqrt{\nu_{-} v_{+}} k_{2}, v_{+} k_{2}\right]$ respectively. More precisely, these two values are the two positive roots of the biquadratic polynomial

$$
p^{4}+\left[v_{-} v_{+}\left(k_{1}^{2}+k_{2}^{2}\right)-k_{1} k_{2}\left(v_{-}+v_{+}\right)^{2}\right] p^{2}+\left(v_{-} v_{+} k_{1} k_{2}\right)^{2} .
$$

Proof We give here the basic outline of the proof, the details can be found in [5]. Drawing graphs helps visualizing the proof. It was already shown that $p$ can be restricted to the interval $\left[v_{-} k_{1}, v_{+} k_{2}\right]$ in Lemma 1 . The main idea is to look separately at three different subintervals for $p$,

$$
I_{l}=\left[\nu_{-} k_{1}, \sqrt{\nu_{-} \nu_{+}} k_{1}\right], \quad I_{c}=\left[\sqrt{\nu_{-} \nu_{+}} k_{1}, \sqrt{\nu_{-} \nu_{+}} k_{2}\right], \quad I_{r}=\left[\sqrt{\nu_{-} \nu_{+}} k_{2}, v_{+} k_{2}\right] .
$$

We have that $k_{c}(p)=\left(v_{-} v_{+}\right)^{-\frac{1}{2}} p$ (the interior local maximum) lies in the interval $\left[k_{1}, k_{2}\right]$ only when $p \in I_{c}$. In this subinterval, we can show using a straightforward argument that the minimal convergence factor is attained when we make the value at $k_{1}$ and $k_{2}$ the same (equioscillation at the endpoints). This gives the parameter value $p_{c}=\sqrt{\nu_{-} \nu_{+} k_{1} k_{2}}$. Note that it might happen that the value at the interior local maximum $R_{c}$ is greater than the value at the endpoints when $p=p_{c}$ (at equioscillation). In that case, we see that moving the parameter $p$ in an interval around $p_{c}$ still yields the same minimum value given by $R_{c}$. 
Now, it remains to look at values of $p$ in the intervals $I_{l}$ and $I_{r}$, when the local maximum $k_{c}$ lies outside the range for $k$, and compare the result with $p_{c}$. The situation in these two intervals is perfectly symmetric and it is sufficient to consider only one of those, say $p \in I_{r}$. When $p=\sqrt{\nu_{-} \nu_{+}} k_{2}$, we have $k_{c}(p)=k_{2}$. For this value of $p$ the convergence factor at the endpoints is

$$
\rho\left(k_{1}, p\right)=\frac{\left(\sqrt{\mu} k_{r}-1\right)\left|k_{r}-\sqrt{\mu}\right|}{\left(\sqrt{\mu} k_{r}+1\right)\left(k_{r}+\sqrt{\mu}\right)}=: R_{\text {ext }}, \quad \rho\left(k_{2}, p\right)=\rho\left(k_{c}(p), p\right)=R_{c} .
$$

The argument then splits into two separate cases.

(i) If $k_{r} \geq f(\mu)$, we have that $R_{\text {ext }} \geq R_{c}$. Because $\rho\left(k_{1}, p\right)$ increases as we increase $p$, we cannot improve the convergence factor for $p>\sqrt{\nu_{-} \nu_{+}} k_{2}$. We also note that $R_{\text {ext }} \geq \max _{k} \rho\left(k, p_{c}\right)$, thus we can take $p=p_{c}$ defined above as a minimizer.

(ii) If $k_{r}<f(\mu)$, we get $R_{\text {ext }}<R_{c}$ and we can find a value $p_{r} \in I_{r}$ such that

$$
\rho\left(k_{1}, p_{r}\right)=\rho\left(k_{2}, p_{r}\right)<R_{c},
$$

which beats the best convergence factor that can be obtained for $p \in I_{c}$. By the same argument, we can show there is also a value $p_{l} \in I_{l}$ that satisfies the above equation (with $\rho\left(k_{1}, p_{l}\right)=\rho\left(k_{1}, p_{r}\right)$ ). So, we get two distinct minimizers $p_{r}$ and $p_{l}$, which can be computed by finding the two positive roots of the biquadratic polynomial shown in the theorem statement. Explicit formulas for $p_{r}$ and $p_{l}$ can easily be written down.

We considered all possible scenarios, so this completes the proof.

Figure 1 shows four possible behaviors of the optimized convergence factor, depending on how the ratio of coefficients $\mu$ compares to the ratio of frequencies $k_{r}$. In the upper-right and bottom-left graphs, any $p$ in an interval attains the same minimum, because $R_{c}$ is fixed. In the bottom-right graph, we are in the case where two distinct values of $p\left(p_{l}\right.$ and $\left.p_{r}\right)$ minimize the convergence factor.

Theorem 3 (Asymptotic performance) When $v_{1}$ and $v_{2}$ are kept constant, $k_{2}=\frac{\pi}{h}$ and $h$ is small enough, the optimized Robin parameter given by Theorem 2 is $p^{*}=$ $\sqrt{\nu_{-} \nu_{+} k_{1} \pi} h^{-1 / 2}$, and the asymptotic convergence factor of the Schwarz method is

$$
\max _{k_{1} \leq k \leq \pi / h}\left|\rho\left(k, p^{*}\right)\right|=1-2\left(\sqrt{\mu}+\frac{1}{\sqrt{\mu}}\right)\left(\frac{k_{1} h}{\pi}\right)^{\frac{1}{2}}+O(h) .
$$

Proof For $k_{2}$ large enough, we get $k_{r}>f(\mu)$, and so $p^{*}=\sqrt{\nu_{-} \nu_{+} k_{1} \pi} h^{-1 / 2}$ is a minimizer. Also, $\rho\left(k_{1}, p^{*}\right)$ approaches 1 as $h \rightarrow 0$, and $R_{c}$ is a constant. Thus, eventually (for $h$ small enough) we have $\rho\left(k_{1}, p^{*}\right)>R_{c}$ and the minimizer $p^{*}$ is unique. Finally, expanding $\rho\left(k_{1}, p^{*}\right)$ for small $h$ gives the stated result. 

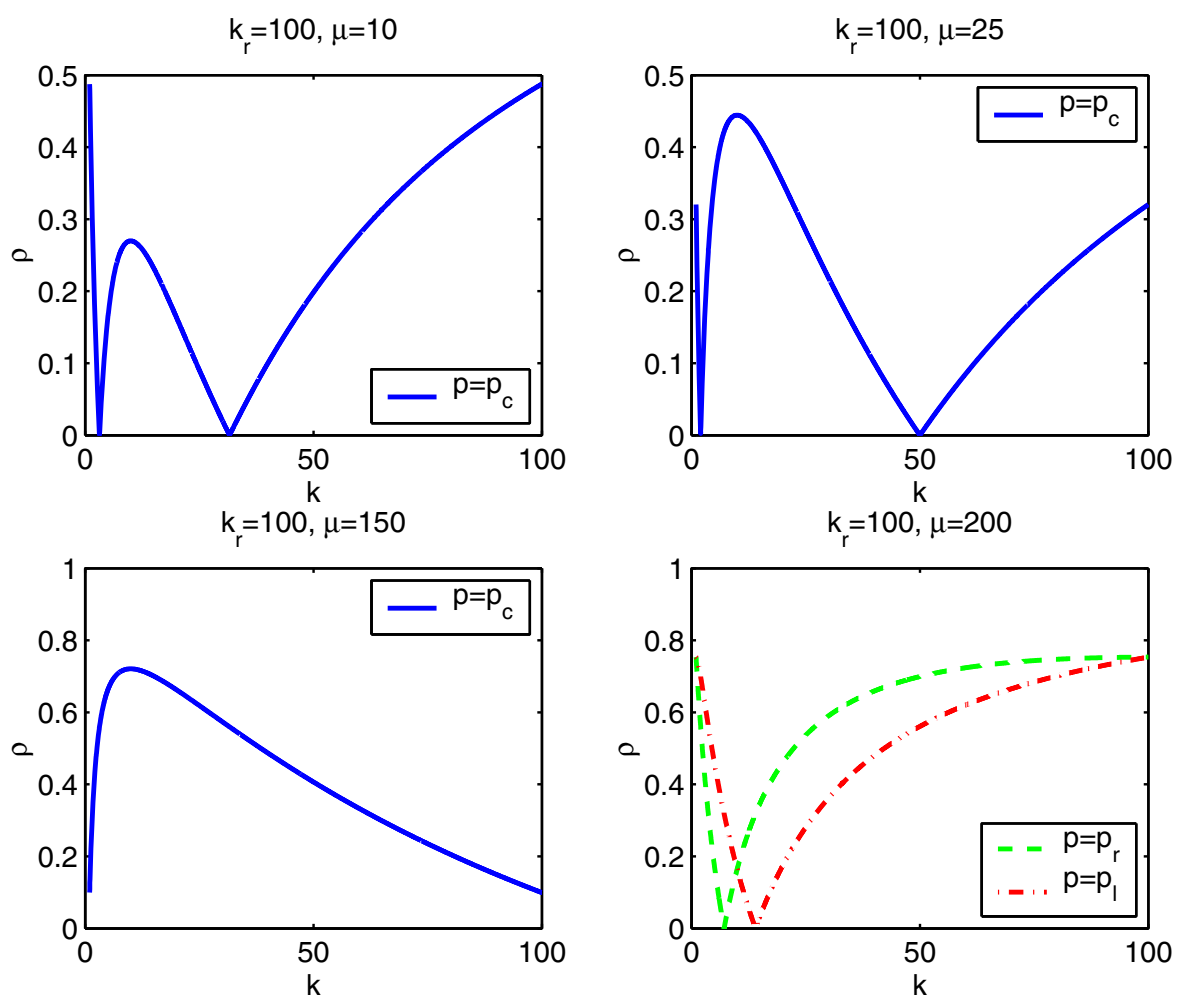

Fig. 1 Optimized convergence factor for different ratios of coefficients

By setting $v_{1}=v_{2}=: v$ in the above two theorems, we recover the results in the case of constant coefficient, in agreement with [11], namely that

$$
p^{*}=v \sqrt{k_{1} k_{2}}, \quad \max _{k_{1} \leq k \leq \pi / h}\left|\rho\left(k, p^{*}\right)\right|=1-4 \sqrt{k_{1}} \pi h^{\frac{1}{2}}+O(h) .
$$

\subsection{Optimized Robin conditions: second version}

From Fig. 1, it can be noticed that the optimized convergence factor is not very good when the ratio of coefficients, $\mu$, is large. This can be justified by the fact that it is not appropriate to approximate the optimal symbols $\sigma_{1}^{o p t}=v_{2}|k|$ and $\sigma_{2}^{o p t}=v_{1}|k|$ with the same parameter value when $v_{1}$ and $v_{2}$ are very different.

There is a second choice that we can make, which is directly driven by the form of the optimal symbols. To be consistent with these symbols, we now use a different scaling of the Robin parameters,

$$
\sigma_{1}(k)=v_{2} q, \quad \sigma_{2}(k)=v_{1} q, \quad \text { for } q \in \mathbb{R} .
$$


In this case, the convergence factor can be written, for $k>0$, as

$$
\rho(k, q)=\frac{(q-k)^{2}}{(q+\mu k)(q+k / \mu)},
$$

where $\mu:=\frac{v_{+}}{v_{-}}$as before. The condition $q>0$ will again be sufficient to guarantee convergence, by Theorem 1. The min-max problem we wish to solve to find the optimized value for $q$ is

$$
\min _{q \in \mathbb{R}}\left(\max _{k_{1} \leq k \leq k_{2}} \rho(k, q)\right) .
$$

It turns out that this optimization problem is a lot easier to solve compared to $\left(M_{1}\right)$, and more importantly it leads to a better convergence factor.

Theorem 4 (Optimized Robin parameter: second version) The unique optimized Robin parameter $q^{*}$ solving the min-max problem $\left(M_{2}\right)$ is given by $q^{*}=\sqrt{k_{1} k_{2}}$.

Proof By taking the derivative of the convergence factor with respect to $q$ and $k$ respectively we find

$$
\operatorname{sign}\left(\frac{\partial \rho}{\partial q}\right)=\operatorname{sign}(q-k), \quad \operatorname{sign}\left(\frac{\partial \rho}{\partial k}\right)=\operatorname{sign}(k-q) .
$$

From these facts, we deduce the following properties.

(i) We can restrict the range of $q$ to the interval $\left[k_{1}, k_{2}\right]$.

(ii) The convergence factor is decreasing in $k$ on $\left(k_{1}, q\right)$, and strictly increasing on $\left(q, k_{2}\right)$.

(iii) $\rho\left(k_{1}, q\right)$ is increasing with respect to $q$, and $\rho\left(k_{1}, k_{1}\right)=0$.

(iv) $\rho\left(k_{2}, q\right)$ is decreasing with respect to $q$, and $\rho\left(k_{2}, k_{2}\right)=0$.

We can thus conclude that we minimize the convergence factor uniformly when its value at $k_{1}$ and $k_{2}$ are equal, $\rho\left(k_{1}, q^{*}\right)=\rho\left(k_{2}, q^{*}\right)$ (equioscillation). Solving this equation for $q^{*}$, we find $q^{*}=\sqrt{k_{1} k_{2}}$.

Theorem 5 (Asymptotic performance) When $v_{1}$ and $v_{2}$ are kept constant, $k_{2}=\frac{\pi}{h}$ and $h$ goes to 0 , the optimized Robin parameter given by Theorem 4 is $q^{*}=$ $\sqrt{k_{1} \pi} h^{-\frac{1}{2}}$ and the asymptotic convergence factor of the Schwarz iteration is

$$
\max _{k_{1} \leq k \leq \frac{\pi}{h}} \rho\left(k, q^{*}\right)=1-\frac{(\mu+1)^{2}}{\mu}\left(\frac{k_{1} h}{\pi}\right)^{\frac{1}{2}}+O(h) .
$$

Proof The result is obtained by simply expanding $\rho\left(k_{1}, q^{*}\right)$ for small values of $h$.

Again, when we set $v_{1}=v_{2}=: v$ in the above two theorems, we also get the appropriate optimized conditions for the case of continuous diffusion coefficient, exactly as in (8).

We now proceed to show that, asymptotically, this second choice of scaling of the Robin parameters is better than the first one studied in Section 3.1. By comparing (7) 

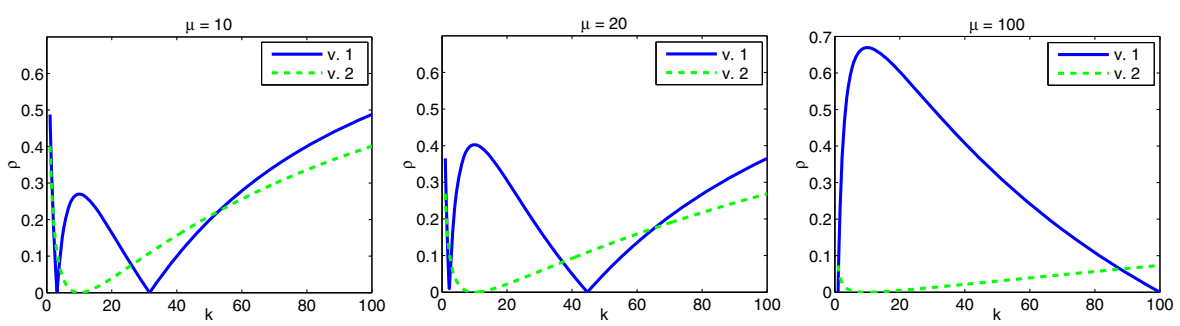

Fig. 2 Comparison of the convergence factors for the two versions of optimized Robin conditions. Here, $v_{2}=k_{1}=1, k_{2}=100$, and only $v_{1}$ is varied

and (9), we see that both choices give similar asymptotic convergence factors, in the form $1-C h^{1 / 2}$, but with different constants $C$. For comparison, the constants for the first and second choice of Robin conditions are respectively

$$
C_{1}=2 \frac{\left(v_{1}+v_{2}\right)}{\sqrt{\nu_{1} \nu_{2}}} \sqrt{\frac{k_{1}}{\pi}} \quad \text { and } \quad C_{2}=\frac{\left(v_{1}+v_{2}\right)^{2}}{v_{1} v_{2}} \sqrt{\frac{k_{1}}{\pi}} .
$$

Noting that $2 x \leq x^{2}$ when $x \geq 2$, and that in this case $x=\frac{\left(v_{1}+v_{2}\right)}{\sqrt{v_{1} v_{2}}} \geq 2$ for any $v_{1}, v_{2}>0$, we find $C_{1} \leq C_{2}$, with equality only when $v_{1}=v_{2}$. Thus, this shows that the second version of optimized Robin conditions yields a better (larger) constant in the asymptotic convergence factor. In practice, it seems that the optimized convergence factor for the second version is always smaller than for the first version, not only asymptotically.

Figure 2 shows a comparison of the optimized convergence factors, when using each choice of Robin conditions studied so far. When the ratio of coefficients is large, the second version yields a much smaller convergence factor. We will provide theoretical justification of this observation in Section 3.6, and confirm it with numerical experiments as well.

\subsection{Optimized two-sided Robin conditions}

Let us now consider the most general Robin conditions, with two free parameters

$$
\sigma_{1}(k)=v_{2} p, \quad \sigma_{2}(k)=v_{1} q, \quad \text { with } p, q \in \mathbb{R} .
$$

Again, we have scaled $\sigma_{j}$ consistently with the optimal symbols, but here it is only to simplify the calculations; it does not matter since $p$ and $q$ are two independent free parameters.

Let $\lambda:=\frac{v_{1}}{v_{2}}$ (not to be confused with $\mu$, which is always greater than 1 ). The convergence factor can be written as

$$
\rho(k, p, q)=\left|\frac{(p-k)(q-k)}{(p+\lambda k)(q+k / \lambda)}\right| .
$$

Before finding optimized values, let us first state sufficient (but not necessary) conditions on $p$ and $q$ to obtain convergence of the iteration, using Theorem 1 . 
Corollary 1 Suppose the Robin parameters $p, q \in \mathbb{R}$ satisfy the orderings

$$
0<q \leq p \text { when } v_{1}<v_{2}, \quad \text { or } 0<p \leq q \text { when } v_{2}<v_{1} .
$$

Then, we have $\rho(k, p, q)<1$ for all $k>0$.

The min-max problem to solve for optimized two-sided Robin conditions is

$$
\min _{p, q \in \mathbb{R}}\left(\max _{k_{1} \leq k \leq k_{2}} \rho(k, p, q)\right) .
$$

We will solve this optimization problem and show that the unique optimized parameters $p^{*}$ and $q^{*}$ satisfy an equioscillation property of the convergence factor. In the process, we will show that the optimized parameters statisfy the conditions of Corollary 1, hence guaranteeing convergence. We break down the proof into a sequence of lemmas, but the main steps are the same as in the previous sections, namely

(1) restrict the range for the parameters,

(2) locate potential candidates for local maxima in $k$,

(3) analyze how these local maxima behave when varying the parameters.

Lemma 3 It is sufficient to consider only positive values for the parameters $p$ and $q$, i.e. the min-max problem $\left(M_{3}\right)$ is equivalent to the problem

$$
\min _{p, q>0}\left(\max _{k_{1} \leq k \leq k_{2}} \rho(k, p, q)\right) .
$$

Proof For $p<0$, it is easy to see that $\rho(k, p, q)>\rho(k,-p, q)$. Similarly, for $q<0, \rho(k, p, q)>\rho(k, p,-q)$. Moreover, $\frac{\partial \rho}{\partial p}(k, 0, q)<0$ and $\frac{\partial \rho}{\partial q}(k, p, 0)<0$ for all $k>0$, thus excluding also the values $p=0$ and $q=0$.

Lemma 4 If $\lambda>1$, then $\left(M_{3}\right)$ is equivalent to the problem

$$
\min _{0<p \leq q}\left(\max _{k_{1} \leq k \leq k_{2}} \rho(k, p, q)\right) .
$$

If $\lambda<1$, then $\left(M_{3}\right)$ is equivalent to the problem

$$
\min _{0<q \leq p}\left(\max _{k_{1} \leq k \leq k_{2}} \rho(k, p, q)\right) .
$$

Without loss of generality, we can look only at the min-max problem for the case $\lambda>1$ : the other case $(\lambda<1)$ reduces to the first one by interchanging $p$ and $q$ and replacing $\lambda$ by $1 / \lambda$.

Proof Suppose $\lambda>1$ and $p>q$. Then,

$$
\rho(k, p, q)=\frac{\left|k^{2}-(p+q) k+p q\right|}{\left|k^{2}+(p / \lambda+\lambda q) k+p q\right|} .
$$


By interchanging the values of $p$ and $q$, the numerator of the fraction is unchanged, but the denominator is increased, since

$$
\frac{p}{\lambda}+\lambda q<\frac{q}{\lambda}+\lambda p \quad \text { when } p>q \text { and } \lambda>1
$$

Hence, in this case, $\rho(k, p, q)>\rho(k, q, p)$, i.e. the convergence factor is uniformly improved by interchanging $p$ and $q$. Thus, it is sufficient to consider pairs of parameters such that $p \leq q$. The case when $\lambda<1$ is treated the same way.

Lemma 4 implies that the smaller of the two parameters is associated with the subdomain having the larger diffusion coefficient (e.g. $p<q$ when $v_{2}<v_{1}$ ). At this point, note that the conditions of Corollary 1 are now satisfied. From now on, we assume without loss of generality that $\lambda>1$, and consequently that $p \leq q$.

Lemma 5 We can restrict the range of the parameters $p$ and $q$ to the interval $\left[k_{1}, k_{2}\right]$ : when $\lambda>1$, the min-max problem $\left(M_{3}\right)$ is equivalent to the problem

$$
\min _{k_{1} \leq p \leq q \leq k_{2}}\left(\max _{k_{1} \leq k \leq k_{2}} \rho(k, p, q)\right) .
$$

Proof Taking partial derivatives with respect to the parameters, and analyzing the sign, we find

$$
\begin{aligned}
& \operatorname{sign}\left(\frac{\partial \rho}{\partial p}\right)=\left\{\begin{array}{l}
\text { strictly positive when } k<p, \\
\text { strictly negative when } k>p,
\end{array}\right. \\
& \operatorname{sign}\left(\frac{\partial \rho}{\partial q}\right)=\left\{\begin{array}{l}
\text { strictly positive when } k<q, \\
\text { strictly negative when } k>q .
\end{array}\right.
\end{aligned}
$$

Thus, when $p<k_{1}$, increasing $p$ improves uniformly the convergence factor. When $p>k_{2}$, decreasing $p$ improves uniformly the convergence factor. The same argument holds for $q$.

Lemma 6 (Local maxima in $k$ )

$$
\max _{k_{1} \leq k \leq k_{2}} \rho(k, p, q)=\max \left\{\rho\left(k_{1}, p, q\right), \rho(\sqrt{p q}, p, q), \rho\left(k_{2}, p, q\right)\right\} .
$$

Proof Analyzing the derivative of $\rho$ with respect to $k$, we get that

$$
\operatorname{sign}\left(\frac{\partial \rho}{\partial k}\right)= \begin{cases}-\operatorname{sign}\left(p q-k^{2}\right) & \text { when } k \notin[p, q], \\ \operatorname{sign}\left(p q-k^{2}\right) & \text { when } k \in[p, q],\end{cases}
$$

which implies that

$$
\rho(k, p, q) \text { is }\left\{\begin{array}{l}
\text { strictly decreasing for } k \in\left[k_{1}, p\right) \cup(\sqrt{p q}, q), \\
\text { strictly increasing for } k \in(p, \sqrt{p q}) \cup k \in\left(q, k_{2}\right] .
\end{array}\right.
$$

Thus, the convergence factor $\rho(k, p, q)$ has a local maximum at $k=\sqrt{p q}$.

Figure 3 illustrates this result. Our aim is to show that the optimized parameters $p^{*}$ and $q^{*}$ are obtained by an equioscillation of these three local maxima. We first start by showing the equioscillation at the two endpoints. 


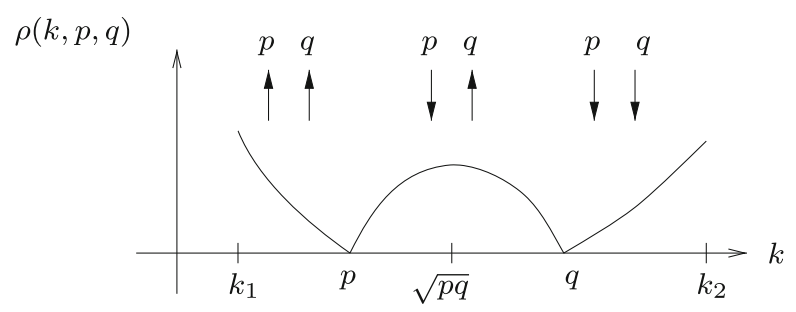

Fig. 3 A sketch of how the convergence factor $\rho(k, p, q)$ behaves as a function of $k$

Lemma 7 (Equioscillation at the endpoints) The optimized convergence factor $\rho\left(k, p^{*}, q^{*}\right)$ must satisfy equioscillation at the endpoints, i.e.

$$
\rho\left(k_{1}, p^{*}, q^{*}\right)=\rho\left(k_{2}, p^{*}, q^{*}\right),
$$

which holds if and only if $p^{*} q^{*}=k_{1} k_{2}$.

Proof We look again at the behavior of $\rho(k, p, q)$ as we vary the parameters. The partial derivatives were already computed in the proof of Lemma 5. From those we can deduce the behavior shown in Fig. 3: the arrows below $p$ and $q$ indicate the change in $\rho(k, p, q)$ (whether it is increasing or decreasing) when $p$ and $q$ are increased, independently.

Let us compare the values of the convergence factor at the endpoints. Suppose first that $\rho\left(k_{1}, p, q\right)<\rho\left(k_{2}, p, q\right)$. Then, from the Fig. 3 we can observe that increasing $p$ uniformly improves $\rho$. In the other case, when we have $\rho\left(k_{1}, p, q\right)>\rho\left(k_{2}, p, q\right)$, decreasing $q$ uniformly improves the convergence factor. One can also easily check that these operations can always be done while staying inside the allowed range for the parameters.

Thus, at the optimized parameters $p^{*}$ and $q^{*}$, we must have $\rho\left(k_{1}, p^{*}, q^{*}\right)=$ $\rho\left(k_{2}, p^{*}, q^{*}\right)$. Some additional algebraic manipulations of this identity lead to the equation $p^{*} q^{*}=k_{1} k_{2}$.

As a consequence of this theorem, the optimized parameters must satisfy

$$
k_{1} \leq p^{*} \leq \sqrt{k_{1} k_{2}} \leq q^{*} \leq k_{2} .
$$

We now have enough tools to complete the proof of the main result.

Theorem 6 (Optimized two-sided Robin parameters) When $\lambda>1$, the unique minimizing pair $\left(p^{*}, q^{*}\right)$ of problem $\left(M_{3}\right)$ is the unique solution of the system of equations

$$
\begin{aligned}
p^{*} q^{*} & =k_{1} k_{2}, \\
\rho\left(k_{1}, p^{*}, q^{*}\right) & =\rho\left(\sqrt{p^{*} q^{*}}, p^{*}, q^{*}\right),
\end{aligned}
$$

such that $p^{*} \leq q^{*}$. 
Proof Lemma 7 directly implies Eq. (11), so we set $q=k_{1} k_{2} p^{-1}$ and note that in this situation $\rho\left(k_{1}, p, q\right)=\rho\left(k_{2}, p, q\right)$. Thus we have reduced the optimization problem $\left(M_{3}\right)$ to solving a one-parameter min-max problem

$$
\min _{k_{1} \leq p \leq \sqrt{k_{1} k_{2}}}\left(\max \left\{R_{1}(p), R_{c}(p)\right\}\right)
$$

where we now use the shorthand notation

$$
R_{1}(p):=\rho\left(k_{1}, p, \frac{k_{1} k_{2}}{p}\right), \quad R_{c}(p):=\rho\left(\sqrt{k_{1} k_{2}}, p, \frac{k_{1} k_{2}}{p}\right) .
$$

It remains to show that the unique solution of problem $\left(\hat{M}_{3}\right)$ occurs when $R_{1}\left(p^{*}\right)=$ $R_{c}\left(p^{*}\right)$. By direct computation, we find that

$$
\operatorname{sign}\left(\frac{d R_{c}}{d p}\right)=\operatorname{sign}\left(p-\sqrt{k_{1} k_{2}}\right)<0, \quad \forall p \in\left[k_{1}, \sqrt{k_{1} k_{2}}\right),
$$

so the value of $R_{c}(p)$ is strictly decreasing in $p$.

Now, expanding the expression for $R_{1}(p)$, we can write

$$
R_{1}(p)=\frac{k_{1}^{2}+k_{1} k_{2}-\left(p+\frac{k_{1} k_{2}}{p}\right) k_{1}}{k_{1}^{2}+k_{1} k_{2}+\left(\frac{p}{\lambda}+\lambda \frac{k_{1} k_{2}}{p}\right) k_{1}}=: \frac{[N]}{[D]},
$$

where the numerator $[N]$ and denominator $[D]$ of the fraction are nonnegative. Taking a derivative in $p$ (using the quotient rule), we get

$$
\frac{d R_{1}}{d p}=\frac{k_{1}}{p^{2}}\left[\left(k_{1} k_{2}-p^{2}\right)[D]+\frac{1}{\lambda}\left(\lambda^{2} k_{1} k_{2}-p^{2}\right)[N]\right] /[D]^{2} .
$$

Examining both terms in the top of the fraction, we see they are both nonnegative since, using the assumption that $\lambda>1$,

$$
\lambda^{2} k_{1} k_{2}>k_{1} k_{2}=p q \geq p^{2} .
$$

Thus, $R_{1}(p)$ is strictly increasing in $p$ on the interval $\left[k_{1}, \sqrt{k_{1} k_{2}}\right)$.

In addition, at the extremal values of $p$, we have

$$
\begin{aligned}
& 0=R_{1}\left(k_{1}\right)<R_{c}\left(k_{1}\right), \\
& R_{1}\left(\sqrt{k_{1} k_{2}}\right)>R_{c}\left(\sqrt{k_{1} k_{2}}\right)=0 .
\end{aligned}
$$

These facts are sufficient to conclude that there exists a unique $p^{*}$ in the interval $\left(k_{1}, \sqrt{k_{1}, k_{2}}\right)$ such that $R_{1}\left(p^{*}\right)=R_{c}\left(p^{*}\right)$, giving the unique solution to the optimization problem $\left(M_{3}\right)$.

Theorem 6 states that the unique pair of optimized parameters $\left(p^{*}, q^{*}\right)$ can be found by solving the system of nonlinear equations (11)-(12). However, some extra algebraic manipulations allow us to show that computing $p^{*}$ reduces to the task of finding the unique real root, in the interval $\left(k_{1}, \sqrt{k_{1} k_{2}}\right)$, of the quartic

$$
\left(p+\lambda k_{1}\right)\left(p+\lambda k_{2}\right)\left(\sqrt{k_{1} k_{2}}-p\right)^{2}-\left(p-k_{1}\right)\left(k_{2}-p\right)\left(p+\lambda \sqrt{k_{1} k_{2}}\right)^{2}=0 .
$$


There exist explicit formulas for writing down the roots of this quartic, but they are fairly complex. Instead, a less error-prone method to obtain the optimized parameter $p^{*}$ is to simply apply a nonlinear solver to Eq. (13) instead.

Theorem 7 (Asymptotic performance) When $\lambda=\mu>1$ is fixed, $k_{2}=\frac{\pi}{h}$ and $h$ goes to 0 , the optimized two-sided Robin parameters are

$$
p^{*} \approx \frac{2 \mu k_{1}}{\mu-1}-\frac{4 \mu k_{1}^{3 / 2}}{\sqrt{\pi}} \frac{(\mu+1)^{2}}{(\mu-1)^{3}} h^{\frac{1}{2}}, \quad q^{*} \approx \frac{\pi(\mu-1)}{2 \mu} h^{-1}+\sqrt{k_{1} \pi} \frac{(\mu+1)^{2}}{\mu(\mu-1)} h^{-\frac{1}{2}},
$$

and the asymptotic convergence factor of the Schwarz iteration is

$$
\max _{k_{1} \leq k \leq \frac{\pi}{h}} \rho\left(k, p^{*}, q^{*}\right)=\frac{1}{\mu}-\frac{4(\mu+1)}{\mu(\mu-1)} \sqrt{\frac{k_{1}}{\pi}} h^{\frac{1}{2}}+O(h) .
$$

Proof We make the ansatz $p^{*}=A h^{\alpha}+B h^{\beta}$, for some $\alpha<\beta \in \mathbb{R}$. Replacing the ansatz inside the formula $R_{1}\left(p^{*}\right)=R_{c}\left(p^{*}\right)$, expanding for small $h$ and matching the two dominant terms, we find $\alpha=0, \beta=\frac{1}{2}$, and the asymptotic coefficients

$$
A=\frac{2 \mu k_{1}}{\mu-1}, \quad B=-\frac{4 \mu k_{1}^{3 / 2}}{\sqrt{\pi}} \frac{(\mu+1)^{2}}{(\mu-1)^{3}} .
$$

The asymptotic formula for $q^{*}$ is obtained using the relation $q^{*}=k_{1} k_{2} / p^{*}$. Finally, expanding $R_{1}\left(p^{*}\right)$ for small $h$ with the above values, we find the expansion (14).

Theorems 6 and 7 are stated for the case $\lambda>1$ only. When $\lambda<1$, Lemma 4 shows that, by simply interchanging the roles of $p$ and $q$, and replacing $\lambda$ by $1 / \lambda$, we can still apply these results. More precisely, if $\lambda<1$, we can first compute the optimized parameters $\tilde{p}^{*}$ and $\tilde{q}^{*}$ corresponding to $\tilde{\lambda}=1 / \lambda$, then interchange the parameters, namely use $p^{*}=\tilde{q}^{*}$ and $q^{*}=\tilde{p}^{*}$. The asymptotic expansion of the convergence factor (14) still holds in that case, with $\mu=1 / \lambda$.

In the case $v_{1}=v_{2}$, Theorem 6 does not nicely reduce to the results obtained for the case of continuous diffusion coefficient. The asymptotic convergence factor (14) is no longer valid (it degenerates) as $\mu \rightarrow 1$. In fact, this asymptotic performance is somewhat surprising. From the known results for continuous coefficients, we would have expected an asymptotic expansion of the form $1-O\left(h^{1 / 4}\right)$. Instead, for discontinuous coefficients, we find that, when using two-sided optimized Robin transmission conditions, we get mesh independent convergence: the convergence factor is bounded away from 1 , asymptotically as $h \rightarrow 0$. Hence this optimized Schwarz method converges better for the case of discontinuous coefficients, than for continuous coefficients, it uses the jump to its advantage.

\subsection{Optimized second order conditions}

It is also possible to use transmission conditions which include tangential derivatives along the interface. For example, we now consider the following second order 
transmission conditions

$$
\begin{gathered}
{\left[v_{1} \frac{\partial}{\partial x}+v_{2}\left(p-q \frac{\partial^{2}}{\partial y^{2}}\right)\right] u_{1}^{n+1}=\left[v_{2} \frac{\partial}{\partial x}+v_{2}\left(p-q \frac{\partial^{2}}{\partial y^{2}}\right)\right] u_{2}^{n},} \\
{\left[-v_{2} \frac{\partial}{\partial x}+v_{1}\left(p-q \frac{\partial^{2}}{\partial y^{2}}\right)\right] u_{2}^{n+1}=\left[-v_{1} \frac{\partial}{\partial x}+v_{1}\left(p-q \frac{\partial^{2}}{\partial y^{2}}\right)\right] u_{1}^{n},}
\end{gathered}
$$

at $x=0$. These are one-sided transmission conditions, because the same parameters are used in both directions, and we could easily imagine a two-sided version with four parameters. Note that we have properly scaled these conditions in view of the optimal operators, as in Section 3.2 and 3.3. In Fourier space, we get the corresponding symbols

$$
\sigma_{1}(k)=v_{2}\left(p+q k^{2}\right), \quad \sigma_{2}(k)=v_{1}\left(p+q k^{2}\right) .
$$

This is equivalent to approximating the optimal symbols $\sigma_{j}^{o p t}$ with second order polynomials in $k$. We do not include a first order term, since $\sigma_{j}^{o p t}$ are even functions of $k$ (the underlying differential operator is self-adjoint). The convergence factor in this situation is

$$
\rho(k, p, q)=\frac{\left(p+q k^{2}-k\right)^{2}}{\left(p+q k^{2}+\mu k\right)\left(p+q k^{2}+k / \mu\right)} .
$$

We will assume a priori that the parameters are strictly positive, for simplicity, and look to solve the associated optimization problem

$$
\min _{p, q>0}\left(\max _{k_{1} \leq k \leq k_{2}} \rho(k, p, q)\right) .
$$

Lemma 8 Any minimizing pair $\left(p^{*}, q^{*}\right)$ of problem $\left(M_{4}\right)$ must satisfy the inequality $p^{*} q^{*} \leq \frac{1}{4}$.

Proof Computing partial derivatives with respect to the parameters, we find that

$$
\operatorname{sign}\left(\frac{\partial \rho}{\partial p}\right)=\operatorname{sign}\left(\frac{\partial \rho}{\partial q}\right)=\operatorname{sign}\left(p+q k^{2}-k\right) .
$$

When $p q>\frac{1}{4}$, the roots of the quadratic $p+q k^{2}-k$ are imaginary, hence implying that $\frac{\partial \rho}{\partial p}>0$ and $\frac{\partial \rho}{\partial q}>0$ for all $k>0$. This means that the convergence factor is uniformly reduced by decreasing $p$ or $q$ or both, until $p q \leq \frac{1}{4}$.

When the coefficients are continuous, it is known that the optimization of twosided Robin conditions and one-sided second order conditions are closely related problems, see [11]. The proof of the following result is inspired by this relation.

Theorem 8 The min-max problem $\left(M_{4}\right)$ has a unique minimizing pair $p^{*}$, $q^{*}$ given by the equioscillation of the convergence factor at the frequencies $k_{1}, k_{2}$ and $k_{c}=$ $\sqrt{p / q}$. This gives the formulas

$$
p^{*}=\frac{\left(k_{1} k_{2}\right)^{\frac{3}{4}}}{\sqrt{2\left(k_{1}+k_{2}\right)}}, \quad q^{*}=\frac{1}{\sqrt{2\left(k_{1}+k_{2}\right)}\left(k_{1} k_{2}\right)^{\frac{1}{4}}} .
$$




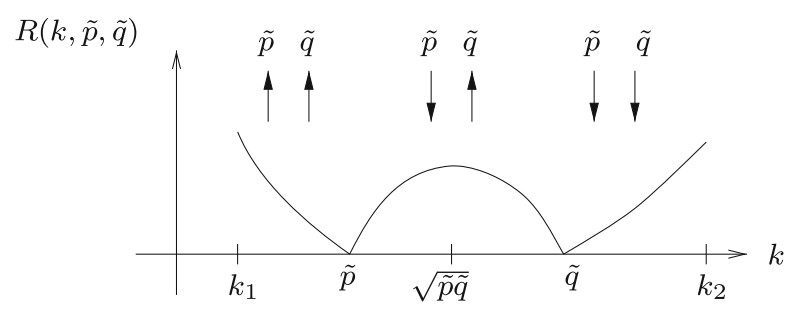

Fig. 4 A sketch of how the convergence factor $R(k, \tilde{p}, \tilde{q})$ behaves as a function of $k$

Proof It will be easier to show equioscillation for a transformed problem. Let us change the parameters $(p, q)$ to $(\tilde{p}, \tilde{q})$, where

$$
\tilde{p}:=\frac{1-\sqrt{1-4 p q}}{2 q}, \quad \tilde{q}:=\frac{1+\sqrt{1-4 p q}}{2 q} .
$$

The new parameters $\tilde{p}$ and $\tilde{q}$ are simply the zeros of the convergence factor $\rho$. This change of variable is well-defined when $p>0, q>0$ and $p q \leq \frac{1}{4}$; the latter inequality was established in Lemma 8 . The inverse transformation of the parameters is given by

$$
p=\frac{\tilde{p} \tilde{q}}{\tilde{p}+\tilde{q}}, \quad q=\frac{1}{\tilde{p}+\tilde{q}} .
$$

The convergence factor in terms of $\tilde{p}$ and $\tilde{q}$ is

$$
R(k, \tilde{p}, \tilde{q}):=\frac{(\tilde{p}-k)^{2}(\tilde{q}-k)^{2}}{\left[\tilde{p} \tilde{q}+k^{2}+\mu(\tilde{p}+\tilde{q})\right]\left[\tilde{p} \tilde{q}+k^{2}+\frac{1}{\mu}(\tilde{p}+\tilde{q})\right]},
$$

and the associated min-max problem is

$$
\min _{0<\tilde{p} \leq \tilde{q}}\left(\max _{k_{1} \leq k \leq k_{2}} R(k, \tilde{p}, \tilde{q})\right) .
$$

This optimization problem looks similar to $\left(M_{3}\right)$, for finding optimized two-sided Robin conditions, but it is in fact significantly different when $\mu \neq 1$. Solving this modified problem with the usual arguments will prove easier than $\left(M_{4}\right)$. Looking at the sign of the various derivatives, we find

$$
\begin{gathered}
\operatorname{sign}\left(\frac{\partial R}{\partial \tilde{p}}\right)=\operatorname{sign}(\tilde{p}-k), \quad \operatorname{sign}\left(\frac{\partial R}{\partial \tilde{q}}\right)=\operatorname{sign}(\tilde{q}-k), \\
\operatorname{sign}\left(\frac{\partial R}{\partial k}\right)=\operatorname{sign}\left((\tilde{p}-k)(k-\tilde{q})\left(\tilde{p} \tilde{q}-k^{2}\right)\right) .
\end{gathered}
$$

The derivatives with respect to the parameters lead us to conclude the bounds

$$
k_{1} \leq \tilde{p} \leq \tilde{q} \leq k_{2} .
$$

Furthermore, taking into account the derivative with respect to $k$, we are able to deduce the behavior shown in Fig. 4, which is exactly the same as the one we obtained for two-sided Robin conditions, in Fig. 3.

Thus, using the same argument as in the proof of Lemma 7, the optimized parameters must satisfy an equioscillation at the endpoints, namely

$$
R\left(k_{1}, \tilde{p}^{*}, \tilde{q}^{*}\right)=R\left(k_{2}, \tilde{p}^{*}, \tilde{q}^{*}\right) .
$$


Solving this algebraic equation for $\tilde{q}$ as a function of $\tilde{p}$, we get three possible formulas:

(1) $\tilde{q}(\tilde{p})=\frac{k_{1} k_{2}}{\tilde{p}}$,

(2) $\tilde{q}(\tilde{p})=x(\tilde{p})+\sqrt{x(\tilde{p})^{2}+k_{1} k_{2}}$ if $\tilde{p}<\sqrt{k_{1} k_{2}}$,

(3) $\tilde{q}(\tilde{p})=\sqrt{x(\tilde{p})^{2}+k_{1} k_{2}}-x(\tilde{p})$ if $\tilde{p}>\sqrt{k_{1} k_{2}}$,

where

$$
x(\tilde{p}):=\frac{\tilde{p}\left(k_{2}-k_{1}\right)^{2}}{2\left|\tilde{p}^{2}-k_{1} k_{2}\right|} .
$$

We now proceed to show that the last two formulas lead to parameter values outside the bounds (16) established earlier.

Suppose first that $k_{1}<\tilde{p}<\sqrt{k_{1} k_{2}}$ and $\tilde{q}(\tilde{p})$ is given by formula (2) above. Then we have the estimate

$$
x(\tilde{p})=\frac{\tilde{p}\left(k_{2}-k_{1}\right)^{2}}{2\left(k_{1} k_{2}-\tilde{p}^{2}\right)}>\frac{\left(k_{2}-k_{1}\right)}{2} .
$$

Substituting this lower bound for $x$ into the formula (2) above, we find that $\tilde{q}(\tilde{p})>$ $k_{2}$, hence $(\tilde{p}, \tilde{q}(\tilde{p}))$ can't be minimizers.

Now suppose that $\sqrt{k_{1} k_{2}}<\tilde{p}<k_{2}$ and $\tilde{q}(\tilde{p})$ is given by formula (3). Then, we find

$$
\frac{\partial x}{\partial \tilde{p}}=-\frac{\left(k_{2}-k_{1}\right)^{2}\left(1+\frac{k_{1} k_{2}}{\tilde{p}^{2}}\right)}{2\left(\tilde{p}-\frac{k_{1} k_{2}}{\tilde{p}}\right)^{2}}<0, \quad \frac{\partial \tilde{q}}{\partial x}=\frac{x}{\sqrt{x^{2}+k_{1} k_{2}}}-1<0 .
$$

Using the chain rule, we get that $\frac{\partial \tilde{q}}{\partial \tilde{p}}=\frac{\partial \tilde{q}}{\partial x} \cdot \frac{\partial x}{\partial \tilde{p}}>0$. Therefore, we have the bound $\tilde{q}(\tilde{p})<\tilde{q}\left(k_{2}\right)=k_{1}$, and so again this third equation cannot give minimizers of the min-max problem.

The only valid equation remaining is the first one, $\tilde{q}=\frac{k_{1} k_{2}}{\tilde{p}}$. Substituting for $\tilde{q}$ in the convergence factor, we are left with a function of $k$ and $\tilde{p}$ only, $S(k, \tilde{p}):=$ $R\left(k, \tilde{p}, k_{1} k_{2} / \tilde{p}\right)$, and wish to solve the problem

$$
\min _{k_{1} \leq \tilde{p} \leq \sqrt{k_{1} k_{2}}}\left(\max \left\{S\left(k_{1}, \tilde{p}\right), S\left(\sqrt{k_{1} k_{2}}, \tilde{p}\right)\right\}\right) .
$$

It remains to show that this problem is solved by equioscillation of the two values. Analyzing the partial derivative of each of those function values, we get

$$
\begin{aligned}
\operatorname{sign}\left(\frac{\partial S}{\partial \tilde{p}}\left(k_{1}, \tilde{p}\right)\right) & =\operatorname{sign}\left(\left(\tilde{p}-k_{1}\right)\left(\tilde{p}-k_{2}\right)\left(\tilde{p}^{2}-k_{1} k_{2}\right)\right) & >0, \\
\operatorname{sign}\left(\frac{\partial S}{\partial \tilde{p}}\left(\sqrt{k_{1} k_{2}}, \tilde{p}\right)\right) & =\operatorname{sign}\left(\tilde{p}^{2}-k_{1} k_{2}\right) & <0 .
\end{aligned}
$$

Moreover, when inserting the extremal values of the parameter $\tilde{p}$, we get

$$
\begin{aligned}
& 0=S\left(k_{1}, k_{1}\right)<S\left(k_{1}, \sqrt{k_{1} k_{2}}\right), \\
& S\left(\sqrt{k_{1} k_{2}}, k_{1}\right)>S\left(\sqrt{k_{1} k_{2}}, \sqrt{k_{1} k_{2}}\right)=0 .
\end{aligned}
$$

Hence, the unique minimizer of problem (17) is given by the unique parameter $\tilde{p}^{*} \in$ $\left(k_{1}, \sqrt{k_{1} k_{2}}\right)$ such that $S\left(k_{1}, \tilde{p}^{*}\right)=S\left(\sqrt{k_{1} k_{2}}, \tilde{p}^{*}\right)$. Solving this algebraic equation, 
we find that the unique minimizing pair $\left(\tilde{p}^{*}, \tilde{q}^{*}\right)$ for the transformed problem $\left(\tilde{M}_{4}\right)$ is given by the direct formulas

$$
\begin{aligned}
& \tilde{p}^{*}=\frac{\left(k_{1} k_{2}\right)^{\frac{1}{4}}}{\sqrt{2}}\left[\sqrt{k_{1}+k_{2}}-\left(\sqrt{k_{2}}-\sqrt{k_{1}}\right)\right], \\
& \tilde{q}^{*}=\frac{\left(k_{1} k_{2}\right)^{\frac{1}{4}}}{\sqrt{2}}\left[\sqrt{k_{1}+k_{2}}+\left(\sqrt{k_{2}}-\sqrt{k_{1}}\right)\right] .
\end{aligned}
$$

Transforming back to the original parameter space $(p, q)$, we obtain the formulas (15). Note that equioscillation of the transformed convergence factor at $k_{1}, \sqrt{\tilde{p} \tilde{q}}$, and $k_{2}$ is equivalent to the equioscillation of the orginal convergence factor at $k_{1}, \sqrt{\frac{p}{q}}$ and $k_{2}$.

Theorem 9 When $v_{1}$ and $v_{2}$ are fixed, $k_{2}=\frac{\pi}{h}$ and $h$ goes to 0 , the optimized second order parameters behave as

$$
p^{*}=\frac{k_{1}^{\frac{3}{4}} \pi^{\frac{1}{4}}}{\sqrt{2}} h^{-\frac{1}{4}}+O\left(h^{\frac{3}{4}}\right), \quad q^{*}=\frac{1}{\sqrt{2} \pi^{\frac{3}{4}} k_{1}^{\frac{1}{4}}} h^{\frac{3}{4}}+O\left(h^{\frac{7}{4}}\right),
$$

and the asymptotic convergence factor of the Schwarz iteration is

$$
\max _{k_{1} \leq k \leq \pi / h} \rho\left(k, p^{*}, q^{*}\right)=1-\sqrt{2}\left(2+\mu+\frac{1}{\mu}\right)\left(\frac{k_{1}}{\pi}\right)^{\frac{1}{4}} h^{\frac{1}{4}}+O\left(h^{\frac{1}{2}}\right) .
$$

Proof These are readily obtained by expanding the formulas (15) for $p^{*}$ and $q^{*}$, and subsequently the convergence factor $\rho\left(k_{1}, p^{*}, q^{*}\right)$ for small $h$ when $k_{2}=\frac{\pi}{h}$.

It is interesting to note that the formulas for the optimized parameters do not depend on the diffusion coefficients at all, only the resulting convergence factor does. In fact, the optimized parameters are the same as those obtained when the coefficient is continuous, i.e. when solving the problem $-\Delta u=f$ (see [11]). This is also true for the one-sided Robin conditions of Section 3.2. Moreover, when the coefficient is continuous, the asymptotic performance of optimized second order conditions and optimized two-sided Robin conditions are comparable. However, here when the coefficient is discontinuous, the optimized two-sided Robin conditions yield a mesh independent asymptotic performance, whereas the optimized second order conditions do not.

\subsection{Comparison of convergence factors}

Figure 5 shows a comparison of the convergence factors for the four different choices of optimized transmission conditions analyzed previously. For this relatively large value of the mesh size, $h=\frac{\pi}{200}$, the performance of the two-sided Robin conditions and second order conditions are comparable: the optimized convergence factors are 

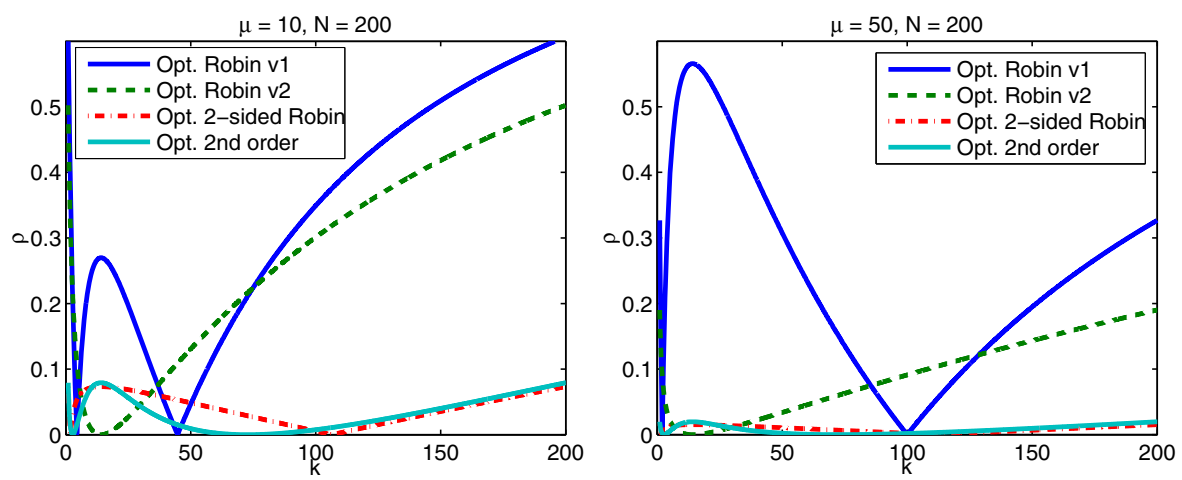

Fig. 5 Convergence factors for $\mu=10$ on the left and $\mu=50$ on the right, in the case $h=\pi / 200$

less than 0.08 when $\mu=10$ and less than 0.02 when $\mu=50$, producing very rapid error reduction.

\subsection{Asymptotics for strong heterogeneity}

All the asymptotic performances derived so far were always with respect to a small grid spacing $h$ while the coefficients $v_{1}, v_{2}$ are held constant. It is also interesting and relevant to consider the case when the ratio of coefficients is very large (corresponding to strong heterogeneity in the material), and how this affects the asymptotics. For this purpose, suppose that $\mu:=\max \left(v_{1}, v_{2}\right) / \min \left(v_{1}, v_{2}\right) \gg 1$, and assume that $h$ is small but fixed.

Theorem 10 (Asymptotics for a large jump in diffusion) For the different optimized conditions, we find different behaviors as $\mu \rightarrow \infty$ and for fixed small $h$. The following expressions are obtained by expanding the optimized convergence factor with respect to large $\mu$, and then keeping only the dominant term for small $h$.

- Optimized Robin conditions, version 1:

$$
\max _{k_{1} \leq k \leq \pi / h}\left|\rho\left(k, p^{*}\right)\right|=\frac{\sqrt{k_{2}}-\sqrt{k_{1}}}{\sqrt{k_{2}}+\sqrt{k_{1}}}+O\left(\frac{1}{\mu}\right) \approx 1-2 \sqrt{\frac{k_{1} h}{\pi}} .
$$

- Optimized Robin conditions, version 2:

$$
\max _{k_{1} \leq k \leq \pi / h}\left|\rho\left(k, q^{*}\right)\right|=\frac{\left(\sqrt{k_{2}}-\sqrt{k_{1}}\right)^{2}}{\sqrt{k_{1} k_{2}}} \cdot \frac{1}{\mu}+O\left(\frac{1}{\mu^{2}}\right) \approx \sqrt{\frac{\pi}{k_{1} h}}\left(\frac{1}{\mu}\right) .
$$

- Optimized two-sided Robin conditions: $p_{0}^{*}:=\lim _{\mu \rightarrow \infty} p^{*}$ and $q_{0}^{*}:=\lim _{\mu \rightarrow \infty} q^{*}$

$$
\max _{k_{1} \leq k \leq \pi / h}\left|\rho\left(k, p^{*}, q^{*}\right)\right|=\frac{\left(p_{0}^{*}-k_{1}\right)\left(q_{0}^{*}-k_{1}\right)}{k_{1} q_{0}^{*}} \cdot \frac{1}{\mu}+O\left(\frac{1}{\mu^{2}}\right) \approx \frac{1}{\mu}
$$


- Optimized second order conditions:

$$
\max _{k_{1} \leq k \leq \pi / h}\left|\rho\left(k, p^{*}, q^{*}\right)\right|=\frac{\left(p^{*}+q^{*} k_{1}^{2}-k_{1}\right)^{2}}{k_{1}\left(p^{*}+q^{*} k_{1}^{2}\right)} \cdot \frac{1}{\mu}+O\left(\frac{1}{\mu^{2}}\right) \approx\left(\frac{\pi}{4 k_{1} h}\right)^{\frac{1}{4}}\left(\frac{1}{\mu}\right) .
$$

Proof For the second version of optimized Robin conditions and for optimized second order conditions, the formulas for the parameters are independent of $\mu$, so it is straightforward to expand the convergence factor as $\mu \rightarrow \infty$.

For the first version of optimized Robin conditions, for $\mu$ large enough we will have $k_{r}<f(\mu)$, and thus fall in the case of two distinct minimizers (see Theorem 2 ). Solving the associated biquadratic, the two roots have the expansions

$$
p_{l}^{*}=\sqrt{k_{1} k_{2}}+\left(k_{2}-k_{1}\right)^{2} \mu^{-1}+O\left(\mu^{-2}\right), \quad p_{r}^{*}=\sqrt{k_{1} k_{2}} \mu-\frac{\left(k_{2}-k_{1}\right)^{2}}{2 \sqrt{k_{1} k_{2}}}+O\left(\mu^{-1}\right) .
$$

When expanding $\rho\left(k_{1}, p^{*}(\mu)\right)$ for large $\mu$, we get the same results for both parameter values.

Finally, for the optimized two-sided Robin conditions, we can deduce that the optimized parameters $p^{*}$ and $q^{*}$ must be constant with respect to $\mu$, to leading order, since both parameters lie in the interval $\left[k_{1}, k_{2}\right]$. In fact, by using the leading order term in the polynomial (13), we find

$$
\begin{aligned}
& p_{0}^{*}=\lim _{\mu \rightarrow \infty} p^{*}=\frac{1}{4}\left(\sqrt{k_{1}}+\sqrt{k_{2}}\right)-\frac{1}{4} \sqrt{\left(\sqrt{k_{1}}+\sqrt{k_{2}}\right)^{4}-16 k_{1} k_{2}}, \\
& q_{0}^{*}=\lim _{\mu \rightarrow \infty} q^{*}=\frac{1}{4}\left(\sqrt{k_{1}}+\sqrt{k_{2}}\right)+\frac{1}{4} \sqrt{\left(\sqrt{k_{1}}+\sqrt{k_{2}}\right)^{4}-16 k_{1} k_{2}} .
\end{aligned}
$$

The result is then obtained by expanding $\rho\left(k_{1}, p_{0}^{*}, q_{0}^{*}\right)$.

The results of this theorem also allow us to classify the performance of the four methods when $\mu$ is very large. For the first version of optimized Robin conditions, the convergence factor does not seem to depend on $\mu$ asymptotically: it approaches a constant close to 1 . For the other three optimized methods that we analyzed, the convergence improves asymptotically for large $\mu$, it behaves like $\rho \approx C(h) \frac{1}{\mu}$. The two-sided Robin conditions have the best constant in front of the leading order term, $C(h)=1$, whereas the one-sided conditions (Robin and second order) have an asymptotic constant $C(h)$ that grows (i.e. gets worse) for small values of $h$.

Now, we can also look at asymptotics when both the jump in the diffusion coefficient is large and the mesh size is small simultaneously. This is relevant, for example, when there could be boundary layers to resolve in the solution, in which case we might be forced to choose the mesh size $h$ as a function of the coefficient ratio, for example we may have a restriction of the form $h \leq C \mu^{-\alpha}$. We show only a specific example of such a combined asymptotic expansion. Suppose that $\nu_{2}=1$ is kept constant and that $\nu_{1}=\varepsilon$ with $\varepsilon \ll 1$.

Proposition 1 (Asymptotics in $h$ and $\varepsilon$ ) For the second version of one-sided optimized Robin conditions, we get three separate cases. 
- If $h$ goes to 0 slower than $\varepsilon^{2}$ (i.e. $\varepsilon h^{-1 / 2} \rightarrow 0$ ),

$$
\max _{k_{1} \leq k \leq \pi / h}\left|\rho\left(k, q^{*}\right)\right| \approx \sqrt{\frac{\pi}{k_{1}}} \varepsilon h^{-1 / 2} .
$$

- If $\varepsilon h^{-1 / 2} \approx C$,

$$
\max _{k_{1} \leq k \leq \pi / h}\left|\rho\left(k, q^{*}\right)\right|=\frac{1}{1+C \sqrt{\frac{k_{1}}{\pi}}}-O\left(h^{1 / 2}\right) .
$$

- If h goes to 0 faster than $\varepsilon^{2}$,

$$
\max _{k_{1} \leq k \leq \pi / h}\left|\rho\left(k, q^{*}\right)\right|=1-\sqrt{\frac{k_{1}}{\pi}} \varepsilon^{-1} h^{1 / 2}+O(h) .
$$

Thus, depending on the asymptotic regime, the convergence factor will keep improving (approaching 0), or will approach a fixed number less than 1, or will deteriorate (approaching 1). For interior layers for problems with discontinuous diffusion coefficient and their discretization, see [8].

\section{The Dirichlet-Neumann method}

Let us look briefly at the Dirichlet-Neumann iterative method, and analyze it in the same framework as the optimized Schwarz methods. In this section, we will consider a diffusion-reaction equation with discontinous coeffcients as a model problem,

$$
\mathcal{L}(u):=-\nabla \cdot(v(x) \nabla u)+c(x) u=0 \quad \text { in } \Omega=\mathbb{R}^{2},
$$

where

$$
v(x)=\left\{\begin{array}{ll}
v_{1} & \text { for } x \in \Omega_{1}, \\
v_{2} & \text { for } x \in \Omega_{2},
\end{array} \quad c(x)= \begin{cases}c_{1} & \text { for } x \in \Omega_{1}, \\
c_{2} & \text { for } x \in \Omega_{2},\end{cases}\right.
$$

with the subdomains $\Omega_{1}=(-\infty, 0) \times \mathbb{R}$ and $\Omega_{2}=(0, \infty) \times \mathbb{R}$.

A first version of the Dirichlet-Neumann method, that we will denote (DN), can be written as

$$
\begin{gathered}
\left\{\begin{aligned}
\mathcal{L}_{1} u_{1}^{n+1}= & 0 \quad \text { in } \Omega_{1}, \\
u_{1}^{n+1}= & \mu^{n} \text { at } x=0,
\end{aligned}\right\}\left\{\begin{array}{cc}
\mathcal{L}_{2} u_{2}^{n+1}=0 & \text { in } \Omega_{2}, \\
v_{2} \frac{\partial u_{2}^{n+1}}{\partial x}=v_{1} \frac{\partial u_{1}^{n+1}}{\partial x} & \text { at } x=0,
\end{array}\right\} \\
\mu^{n+1}=\theta u_{2}^{n+1}+(1-\theta) \mu^{n} \quad \text { at } x=0,
\end{gathered}
$$

where $\theta \in(0,1]$ is a relaxation parameter. A second version, to be denoted (ND), is obtained by imposing the Dirichlet condition in the subdomain $\Omega_{2}$ instead

$$
\begin{gathered}
\left\{\begin{array}{cc}
\mathcal{L}_{1} u_{1}^{n+1}= & 0 \quad \text { in } \Omega_{1}, \\
v_{1} \frac{\partial u_{1}^{n+1}}{\partial x}= & v_{2} \frac{\partial u_{2}^{n+1}}{\partial x} \text { at } x=0,
\end{array}\right\}\left\{\begin{array}{cc}
\mathcal{L}_{2} u_{2}^{n+1}=0 & \text { in } \Omega_{2}, \\
u_{2}^{n+1}=\mu^{n} & \text { at } x=0,
\end{array}\right\} \\
\mu^{n+1}=\theta u_{1}^{n+1}+(1-\theta) \mu^{n} \quad \text { at } x=0 .
\end{gathered}
$$


Note that in both iterations, the Dirichlet problem is solved first. It is also possible to write Dirichlet-Neumann methods in which we relax on the Neumann data instead of the Dirichlet data, but we obtain the same convergence factors as for the corresponding algorithms (DN) or (ND).

For this model problem, with the help of the Fourier transform, we find the convergence factors for the two methods

$$
\begin{aligned}
& \rho_{D N}(k, \theta):=\left|\frac{\hat{u}_{1}^{n+1}(0, k)}{\hat{u}_{1}^{n}(0, k)}\right|=\left|\frac{\hat{u}_{2}^{n+1}(0, k)}{\hat{u}_{2}^{n}(0, k)}\right|=\left|1-\theta\left[1+\frac{v_{1} \lambda^{(1)}(k)}{v_{2} \lambda^{(2)}(k)}\right]\right|, \\
& \rho_{N D}(k, \theta)=\left|1-\theta\left[1+\frac{\nu_{2} \lambda^{(2)}(k)}{v_{1} \lambda^{(1)}(k)}\right]\right|,
\end{aligned}
$$

where $\lambda^{(j)}$ are the characteristic roots of the ordinary differential equation in Fourier space, for each subdomain $\Omega_{j}$, namely $\lambda^{(j)}(k):=\sqrt{k^{2}+\frac{c_{j}}{v_{j}}}$.

It is then natural to ask what is a good value for the relaxation parameter $\theta$ to use. We look for the optimized value by solving the min-max problem

$$
\min _{\theta \in[0,1]}\left(\max _{k_{1} \leq k \leq k_{2}}|\rho(k, \theta)|\right),
$$

for each version of the Dirichlet-Neumann method. First define the functions

$$
F(k):=\frac{v_{1}}{v_{2}} \sqrt{\frac{k^{2}+\frac{c_{1}}{v_{1}}}{k^{2}+\frac{c_{2}}{v_{2}}}}, \quad G(k):=\frac{v_{2}}{v_{1}} \sqrt{\frac{k^{2}+\frac{c_{2}}{v_{2}}}{k^{2}+\frac{c_{1}}{v_{1}}}} .
$$

Theorem 11 (Optimized Relaxation Parameter) The unique minimizers of problem $\left(M_{5}\right)$ for the methods $(D N)$ and $(N D)$ are respectively

$$
\theta_{D N}^{*}=\frac{2}{2+F\left(k_{1}\right)+F\left(k_{2}\right)}, \quad \theta_{N D}^{*}=\frac{2}{2+G\left(k_{1}\right)+G\left(k_{2}\right)} .
$$

Proof One can easily show that the best convergence factor is obtained from the equioscillation at $k=k_{1}$ and $k=k_{2}$.

Theorem 12 (Asymptotic performance of Dirichlet-Neumann) If $\lambda:=\frac{v_{1}}{v_{2}}$ is fixed, $k_{2}=\frac{\pi}{h}$ and $h$ goes to 0 , the asymptotic convergence factor of the Dirichlet-Neumann methods with optimized relaxation parameter is

- for method (DN),

$$
\max _{k_{1} \leq k \leq \pi / h}\left|\rho_{D N}\left(k, \theta_{D N}^{*}\right)\right|=\left|\frac{\lambda-F\left(k_{1}\right)}{2+\lambda+F\left(k_{1}\right)}\right|+O\left(h^{2}\right),
$$

- for method (ND)

$$
\max _{k_{1} \leq k \leq \pi / h}\left|\rho_{N D}\left(k, \theta_{N D}^{*}\right)\right|=\left|\frac{\frac{1}{\lambda}-G\left(k_{1}\right)}{2+\frac{1}{\lambda}+G\left(k_{1}\right)}\right|+O\left(h^{2}\right) .
$$


Thus, the optimized Dirichlet-Neumann methods have mesh independent convergence: the convergence factor approaches a constant strictly less than 1 as $h \rightarrow 0$. Furthermore, when the coefficients are continuous, or when $c_{1}=c_{2}=0$, the optimized relaxation parameters are actually optimal: the convergence factor is uniformly 0 (for both methods). However, when a reaction term is present, the DirichletNeumann cannot be made optimal. Moreover, as soon as we use more subdomains, or the subdomains are of different size, the best relaxation parameter is hard to find; some results can be found in [30].

When using the Dirichlet-Neumann methods without relaxation $(\theta=1)$, one version of the method converges and the other one does not. In that situation, to guarantee convergence, we need to impose the Dirichlet transmission condition for the subproblem that has the smaller diffusion coefficient.

\section{A more general diffusion-reaction model problem}

In this section, we consider the more general problem

$$
\left\{\begin{aligned}
-\nabla \cdot(\mathbf{A}(\mathbf{x}) \nabla u)+c(\mathbf{x}) u & =f \text { for } \mathbf{x} \in \Omega \subseteq \mathbb{R}^{2}, \\
\mathcal{B}(u) & =g \text { for } \mathbf{x} \in \partial \Omega,
\end{aligned}\right.
$$

where $\mathbf{A}(\mathbf{x})$ is a symmetric positive definite matrix and $c(\mathbf{x}) \geq 0$ for all $\mathbf{x} \in \Omega$. This problem allows for anisotropic media, where the diffusion might be different in different directions. We will show that optimized transmission conditions for the Schwarz method for this problem are obtained by solving the same min-max problems that were previously studied for the case of a scalar, isotropic diffusion coefficient $v$.

Suppose the coefficients are piecewise constant on a non-overlapping domain decomposition $\Omega_{1}, \Omega_{2}$ with interface $\Gamma$,

$$
\mathbf{A}(\mathbf{x})=\left\{\begin{array}{ll}
\mathbf{A}^{(1)} & \text { in } \Omega_{1}, \\
\mathbf{A}^{(2)} & \text { in } \Omega_{2},
\end{array} \quad c(\mathbf{x})= \begin{cases}c^{(1)} & \text { in } \Omega_{1}, \\
c^{(2)} & \text { in } \Omega_{2},\end{cases}\right.
$$

where

$$
\mathbf{A}^{(j)}=\left[\begin{array}{ll}
a_{11}^{(j)} & a_{12}^{(j)} \\
a_{12}^{(j)} & a_{22}^{(j)}
\end{array}\right]
$$

are constant, symmetric, positive definite matrices, for $j=1,2$. If we write $u_{j}:=$ $\left.u\right|_{\Omega_{j}}$, then it can be shown (basically using integration by parts) that natural matching conditions across the interface are

$$
\begin{aligned}
& u_{1}=u_{2} \quad \text { on } \Gamma \text {, } \\
& \left(\mathbf{A}^{(1)} \mathbf{n}\right) \cdot \nabla u_{1}=\left(\mathbf{A}^{(2)} \mathbf{n}\right) \cdot \nabla u_{2} \text { on } \Gamma,
\end{aligned}
$$

where $\mathbf{n}$ is the normal vector to $\Gamma$, say pointing outward with respect to $\Omega_{1}$. These are just the conditions of continuity of the solution and of its conormal derivative (or flux).

We analyze a model problem on the domain $\Omega=\mathbb{R}^{2}$ decomposed into the two subdomains $\Omega_{1}=(-\infty, 0) \times \mathbb{R}$ and $\Omega_{2}=(0, \infty) \times \mathbb{R}$, with homogeneous righthand side, $f \equiv 0$. The coupling conditions on the interface $\Gamma=\{x=0\}$ translate 
into

$$
\begin{array}{rlrl}
u_{1} & =u_{2} & \text { at } x & =0, \\
\left(a_{11}^{(1)} \partial_{x}+a_{12}^{(1)} \partial_{y}\right) u_{1} & =\left(a_{11}^{(2)} \partial_{x}+a_{12}^{(2)} \partial_{y}\right) u_{2} \text { at } x=0 .
\end{array}
$$

Using these, we write a consistent general Schwarz iteration in the form

$$
\begin{aligned}
&-\nabla \cdot\left(\mathbf{A}^{(j)} \nabla u_{j}^{n+1}\right)+c^{(j)} u_{j}^{n+1}=0, \quad \text { in } \Omega_{j}, \quad j=1,2, \\
&\left(a_{11}^{(1)} \partial_{x}+a_{12}^{(1)} \partial_{y}+\mathcal{S}_{1}\right) u_{1}^{n+1}=\left(a_{11}^{(2)} \partial_{x}+a_{12}^{(2)} \partial_{y}+\mathcal{S}_{1}\right) u_{2}^{n} \quad \text { at } x=0, \\
&\left(a_{11}^{(2)} \partial_{x}+a_{12}^{(2)} \partial_{y}-\mathcal{S}_{2}\right) u_{2}^{n+1}=\left(a_{11}^{(1)} \partial_{x}+a_{12}^{(1)} \partial_{y}-\mathcal{S}_{2}\right) u_{1}^{n} \quad \text { at } x=0,
\end{aligned}
$$

where $\mathcal{S}_{j}$ are linear operators acting in the tangential direction to the interface, with corresponding Fourier symbols $\sigma_{j}(k)$. Written explicitely, the differential equation we wish to solve is

$$
-a_{11}^{(j)} \frac{\partial^{2} u_{j}}{\partial x^{2}}-a_{22}^{(j)} \frac{\partial^{2} u_{j}}{\partial y^{2}}-2 a_{12}^{(j)} \frac{\partial^{2} u_{j}}{\partial x \partial y}+c^{(j)} u_{j}=0, \quad \text { in } \Omega_{j}, \quad \text { for } j=1,2 .
$$

When taking the Fourier transform of this equation, the characteristic roots we get for the ODE are

$$
\lambda_{ \pm}^{(j)}(k)=\frac{i k a_{12}^{(j)} \pm \sqrt{\left[a_{11}^{(j)} a_{22}^{(j)}-\left(a_{12}^{(j)}\right)^{2}\right] k^{2}+a_{11}^{(j)} c^{(j)}}}{a_{11}^{(j)}} .
$$

Note that $\operatorname{Re}\left(\lambda_{+}^{(j)}\right)>0$ and $\operatorname{Re}\left(\lambda_{-}^{(j)}\right)<0$. To simplify the notation, let

$$
d^{(j)}:=\operatorname{det}\left(\mathbf{A}^{(j)}\right), \quad \gamma^{(j)}(k):=\sqrt{d^{(j)} k^{2}+a_{11}^{(j)} c^{(j)}} .
$$

Using Fourier analysis, we find that the convergence factor for the general Schwarz iteration can be written as

$$
\rho\left(k, \sigma_{1}, \sigma_{2}\right):=\left|\frac{\hat{u}_{j}^{n+1}(0, k)}{\hat{u}_{j}^{n-1}(0, k)}\right|=\left|\frac{\left[\sigma_{1}(k)-\gamma^{(2)}(k)\right]}{\left[\sigma_{1}(k)+\gamma^{(1)}(k)\right]} \cdot \frac{\left[\sigma_{2}(k)-\gamma^{(1)}(k)\right]}{\left[\sigma_{2}(k)+\gamma^{(2)}(k)\right]}\right| .
$$

\subsection{Optimal and optimized Schwarz methods}

From (19), it is clear that optimal symbols are given by

$$
\sigma_{1}^{o p t}(k)=\gamma^{(2)}(k), \quad \sigma_{2}^{o p t}(k)=\gamma^{(1)}(k) .
$$

When there are positive reaction terms, $c^{(j)}>0$, one could use Taylor approximations of order 2 of the optimal symbols in order to get local transmission conditions,

$$
\gamma^{(j)}(k) \approx \sqrt{a_{11}^{(j)} c^{(j)}}+\frac{d^{(j)}}{2 \sqrt{a_{11}^{(j)} c^{(j)}}} k^{2},
$$

but such approximations would only give fast convergence for low frequencies. 
Now, by inspection of the convergence factor (19), we can observe that with the change of variable

$$
v_{j}=\sqrt{d^{(j)}}, \quad \eta_{j}=\frac{a_{11}^{(j)} c^{(j)}}{\sqrt{d^{(j)}}},
$$

we obtain the expression

$$
\rho\left(k, \sigma_{1}, \sigma_{2}\right)=\left|\frac{\left[\sigma_{1}(k)-v_{2} \sqrt{k^{2}+\frac{\eta_{2}}{v_{2}}}\right]}{\left[\sigma_{1}(k)+v_{1} \sqrt{k^{2}+\frac{\eta_{1}}{v_{1}}}\right]} \cdot \frac{\left[\sigma_{2}(k)-v_{1} \sqrt{k^{2}+\frac{\eta_{1}}{v_{1}}}\right]}{\left[\sigma_{2}(k)+v_{2} \sqrt{k^{2}+\frac{\eta_{2}}{v_{2}}}\right]}\right|,
$$

which is exactly the convergence factor of the general Schwarz iteration when applied to the equation

$$
-\nabla \cdot(v(x) \nabla u)+\eta(x) u=0,
$$

with piecewise constant coefficients on the subdomains. Hence, any result that we have for problem (21) can readily be used for the more general problem (18). For example, the min-max problems we need to solve to compute optimized transmission conditions are the same, under the transformation (20). In particular, when $c(x)=0$, the results of Section 3 can be directly applied to compute optimized parameters for problem (18). The same equivalence also applies for the case of continuous coefficients, including the possible use of an overlap, and thus all results found in [11] can be extended to anisotropic diffusions $\mathbf{A}$. When the reaction coefficient $c(x)$ is discontinuous, there are no results available yet for optimized transmission conditions.

\section{Numerical experiments}

The numerical experiments shown in this section were performed on the model problem,

$$
\left\{\begin{aligned}
-\nabla \cdot(v(\mathbf{x}) \nabla u) & =0 & & \text { in } \Omega=(0, \pi) \times(0, \pi), \\
u & =0 & & \text { on } \partial \Omega .
\end{aligned}\right.
$$

The square domain is decomposed into two nonoverlapping subdomains $\Omega_{1}=$ $\left(0, \frac{\pi}{2}\right) \times(0, \pi)$ and $\Omega_{2}=\left(\frac{\pi}{2}, \pi\right) \times(0, \pi)$. We use a finite volume discretization on a uniform grid with grid size $h$. In all the experiments, $v_{1}=\frac{1}{\mu}, v_{2}=1$ where $\mu$ is taken to be larger than 1 . Vectors of random values between -1 and 1 are used as initial guess for the Schwarz iteration so that the initial error includes every possible frequency component. When using the Schwarz method as an iterative solver, exactly as in (2), we use the $L^{\infty}$ error

$$
e^{n}:=\left\|\mathbf{U}-\mathbf{u}^{n}\right\|_{\infty}
$$

where $\mathbf{U}$ is the discrete solution of the global problem (22) and $\mathbf{u}^{n}$ is obtained by combining together the subdomain solutions at iteration $n$, taking the average of $\mathbf{u}_{1}^{n}$ and $\mathbf{u}_{2}^{n}$ on the interface $x=\frac{\pi}{2}$. 


\subsection{Krylov acceleration}

The convergence can be accelerated by using the Schwarz method as a preconditioner for a Krylov subspace method. There are several ways to formulate this; we present in this subsection a specific way which is particularly convenient for implementation.

Suppose we have a domain decomposition into $J$ subdomains. Let us write one iteration of the Schwarz method as

$$
\mathbf{u}^{n+1}=\boldsymbol{\Phi}\left(\mathbf{u}^{n}, \mathbf{f}\right),
$$

where $\mathbf{u}$ is an augmented vector in which the unknowns on the interfaces appear more than once,

$$
\mathbf{u}^{n}=\left[\begin{array}{llll}
\mathbf{u}_{1}^{n} & \mathbf{u}_{2}^{n} \ldots \mathbf{u}_{J}^{n}
\end{array}\right]^{T} .
$$

The function $\boldsymbol{\Phi}$ is linear in each of its arguments, so we can write

$$
\boldsymbol{\Phi}(\mathbf{u}, \mathbf{f})=T \mathbf{u}+N \mathbf{f},
$$

where the matrices $T$ and $N$ are defined by

$$
T \mathbf{u}:=\boldsymbol{\Phi}(\mathbf{u}, \mathbf{0}), \quad N \mathbf{g}:=\boldsymbol{\Phi}(\mathbf{0}, \mathbf{g}),
$$

which are simple applications of a Schwarz iteration with zero right-hand side and zero initial guess respectively. The Schwarz iteration can then be formulated as

$$
\mathbf{u}^{n+1}=\mathbf{u}^{n}+\left(N \mathbf{f}-(I-T) \mathbf{u}^{n}\right),
$$

which is a stationary iterative method for the preconditioned linear system

$$
(I-T) \mathbf{u}=N \mathbf{f} .
$$

To accelerate the convergence, we can use a Krylov subspace method to solve (24) instead. We used BiCGstab instead of GMRES, so that we don't have to choose an appropriate restart parameter and avoid memory problems. Note that the stopping criterion in this case is based on the euclidean norm of the relative residual in the system (24), i.e.

$$
r^{n}=\frac{\left\|N \mathbf{f}-(I-T) \mathbf{u}^{n}\right\|_{2}}{\|N \mathbf{f}\|_{2}} .
$$

\subsection{Comparison of convergence}

In Fig. 6, the convergence of the different methods is shown for two different coefficient ratios. The two Dirichlet-Neumann methods, (DN) and (ND), with optimized relaxation parameters, are converging very quickly. This is because they are optimal methods when using two symmetric subdomains (see Section 4); however their convergence deteriorates very quickly when using more subdomains or when breaking the symmetry (see Section 6.3). Among the other methods presented in Fig. 6, the transmission conditions that were optimized over two free parameters perform much better than the one-sided Robin conditions, as expected. Also, the second version of the one-sided conditions converges faster than the first version with bad scaling, and the difference increases as the ratio of coefficients increases, as predicted. Generally, optimized Schwarz methods converge faster as the jump in the coefficient is increased. 

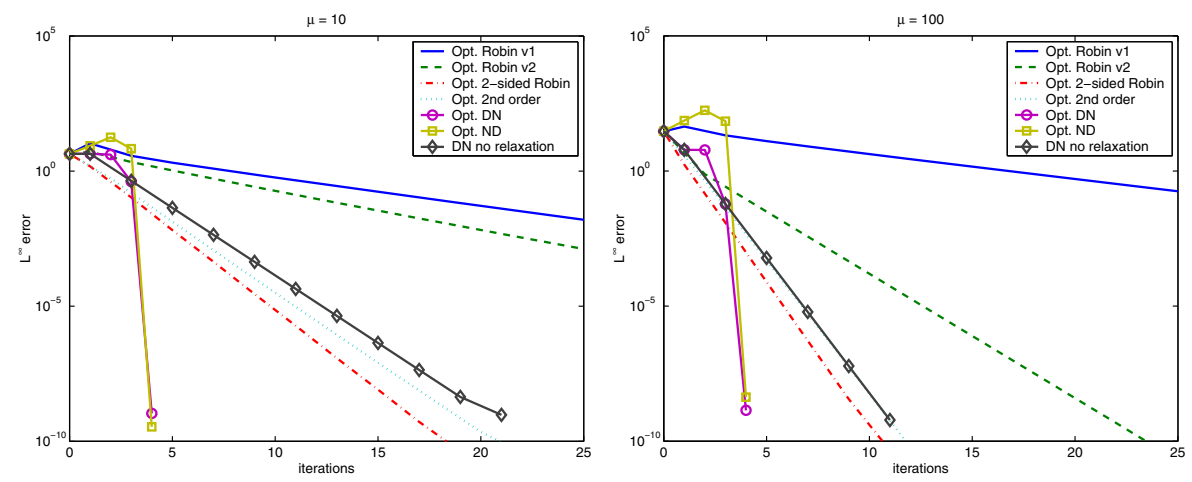

Fig. 6 Convergence for $\mu=10$ on the left and $\mu=100$ on the right, in the case $h=\frac{\pi}{300}$

Next, we fix $\mu=10$ and vary the grid size $h$. Table 1 shows the number of iterations that were needed to reach a tolerance of $10^{-6}$ for the different optimized methods. The convergence of the optimized transmission conditions deteriorates as we decrease the mesh size $h$, except for the two-sided Robin conditions, for which the number of iterations appears to stop growing for small $h$, as the theoretical asymptotics of Section 3 indicated. It is difficult to verify more precisely the asymptotic performances, like it was done in [11], to confirm the exponent of the leading order terms in $h$ : when the coefficient is discontinuous $(\mu>1)$, the expansions we have derived become valid only for very small values of $h$, much smaller values than the ones we could use numerically in Table 1 .

Now, let us fix $h=\frac{\pi}{300}$ and vary the heterogeneity ratio $\mu$. Table 2 shows again the number of iterations that were needed to reach a tolerance of $10^{-6}$ for the different methods. These results show that the first version of the optimized Robin conditions is really not performing well for large discontinuities in the coefficient.

Table 1 Number of iterations to reach a tolerance of $10^{-6}$, for small values of $h$

\begin{tabular}{|c|c|c|c|c|}
\hline & Opt. Robin v.1 & Opt. Robin v.2 & Opt. 2-sided Robin & Opt. 2nd order \\
\hline$h$ & \multicolumn{4}{|c|}{ Optimized Schwarz as an iterative solver } \\
\hline$\frac{\pi}{50}$ & 26 & 24 & 11 & 10 \\
$\frac{\pi}{100}$ & 39 & 30 & 11 & 12 \\
$\frac{\pi}{200}$ & 56 & 40 & 12 & 13 \\
$\frac{\pi}{400}$ & 77 & 54 & 13 & 14 \\
$\frac{\pi}{800}$ & 110 & 73 & 13 & 17 \\
\hline & \multicolumn{4}{|c|}{ Optimized Schwarz with Krylov acceleration } \\
\hline$\frac{\pi}{50}$ & 19 & 13 & 9 & 7 \\
$\frac{\pi}{100}$ & 21 & 14 & 9 & 8 \\
$\frac{\pi}{200}$ & 25 & 17 & 10 & 10 \\
$\frac{\pi}{400}$ & 28 & 21 & 10 & 11 \\
$\frac{\pi}{800}$ & 37 & 26 & 11 & 10 \\
\hline
\end{tabular}


Table 2 Number of iterations to reach a tolerance of $10^{-6}$, for large heterogeneity ratios

\begin{tabular}{|c|c|c|c|c|}
\hline & Opt. Robin v.1 & Opt. Robin v.2 & Opt. 2-sided Robin & Opt. 2nd order \\
\hline$\mu$ & \multicolumn{4}{|c|}{ Optimized Schwarz as an iterative solver } \\
\hline $10^{1}$ & 67 & 48 & 13 & 14 \\
$10^{2}$ & 79 & 16 & 7 & 8 \\
$10^{3}$ & 206 & 9 & 5 & 6 \\
$10^{4}$ & 234 & 7 & 5 & 5 \\
$10^{5}$ & 234 & 5 & 5 & 5 \\
\hline & 29 & 17 & 10 & 9 \\
\hline $10^{1}$ & 29 & 8 & 6 & 6 \\
$10^{2}$ & 59 & 5 & 4 & 4 \\
$10^{3}$ & 50 & 4 & 4 & 3 \\
$10^{4}$ & 41 & 4 & 4 & 4 \\
$10^{5}$ & \multicolumn{4}{|c|}{ Optimized Schwarz with Krylov accceleration } \\
\hline
\end{tabular}

On the other hand, all the other optimized conditions do show significant improvements in the convergence as we increase the ratio $\mu$. This is also in agreement with the theoretical asymptotics presented in Section 3.6. It can be noted, in Table 2, that the convergence of the first version of the optimized Robin conditions seems to show some improvement for large $\mu$, when using Krylov acceleration. However, this may only be due to the fact that the norm used to check convergence depends on the transmission conditions and its parameters. By looking at the convergence history more closely in Fig. 7, we can see that the rate of convergence does not get better as we increase $\mu$.

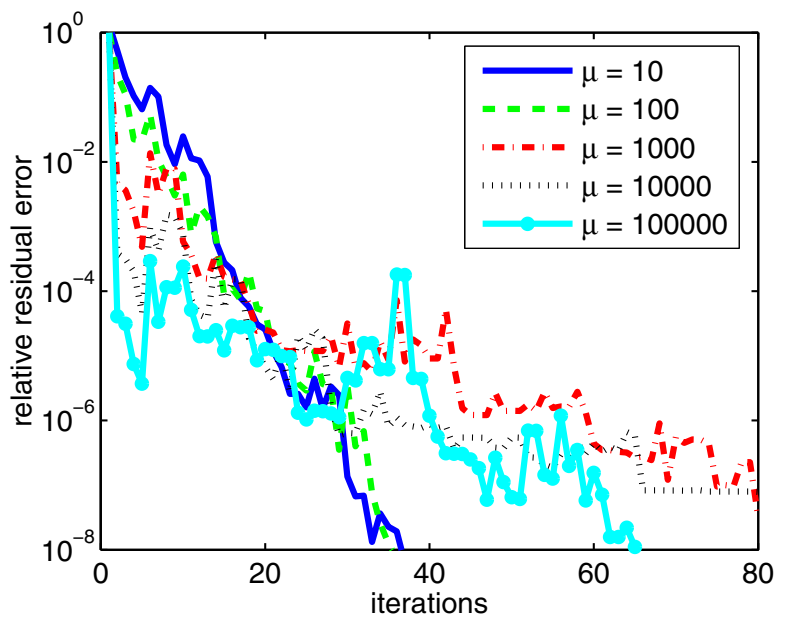

Fig. 7 Convergence of the Schwarz method with optimized Robin conditions (first version of one-sided) when used as a preconditioner for BiCGstab, for the case $h=\frac{\pi}{300}$ 

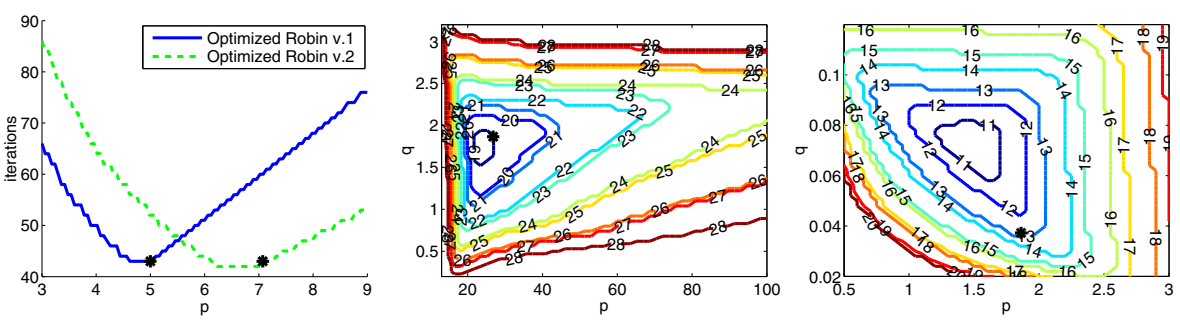

Fig. 8 Optimized parameters (*) computed from the min-max problem (when $\mu=2$ ), compared with the performance of other values of the parameters, on the left for the one-sided Robin conditions, in the middle for the two-sided Robin conditions, and on the right for the second order conditions

Recall that the optimized parameters for the transmission conditions are computed by analyzing a convergence factor that was derived for a continuous model problem on the infinite plane. We now compare these optimized values with the parameters that yield the fastest convergence numerically for the discrete problem, in the case $\mu=2$ and $h=\frac{\pi}{50}$. Figure 8 shows the number of iterations required to reach a tolerance of $10^{-6}$ for a range of parameter values around the optimized parameters. We see that the Fourier analysis performed on the continuous model problem predicts very well the best parameters.

\subsection{Deterioration of convergence}

We should point out that there are extreme situations when this continuous analysis will fail to produce very good parameter values, for example

- when the subdomains are very thin or very asymmetrical,

- when the coefficients of the problem are varying a lot inside the subdomains,

- when the shape of the interface is very far from straight.

In such cases, one should solve modified min-max problems that are better adapted to these situations instead, see for example [21]. In this subsection, we illustrate two scenarios when the convergence deteriorates: with two very asymmetrical subdomains, and with many subdomains.

Recall that the computational domain for the numerical experiments is the square $(0, \pi)^{2}$. We consider now an asymmetrical decomposition into 2 subdomains

$$
\Omega_{1}=(0, \alpha \pi) \times(0, \pi), \quad \Omega_{2}=(\alpha \pi, \pi) \times(0, \pi), \quad \text { where } \alpha \in(0,1) .
$$

Thus, the subdomains are perfectly symmetric when $\alpha=\frac{1}{2}$. Table 3 displays some results when we vary the position of the interface. We also compare with Dirichlet-Neumann methods with optimal relaxation parameters from Section 4. These methods converge very quickly when the two subdomains are symmetric, but the convergence deteriorates as soon as we break the symmetry in the decomposition, and divergence (D) is even observed in some cases. The most stable method across the table is the optimized second order transmission conditions, which does not show a significant change in convergence as the position of the interface is moved. 
Table 3 Number of iterations needed to reach a tolerance of $10^{-6}$, for different values of $\alpha$, when $\boldsymbol{\mu}=\mathbf{2}$, $N=200$

\begin{tabular}{|l|c|c|c|c|c|c|c|}
\hline Interface position $\alpha$ & $\frac{1}{20}$ & $\frac{1}{8}$ & $\frac{1}{4}$ & $\frac{1}{2}$ & $\frac{3}{4}$ & $\frac{7}{8}$ & $\frac{19}{20}$ \\
\hline Opt. Robin v.1 & 65 & 65 & 78 & 87 & 69 & 65 & 65 \\
Opt. Robin v.2 & 63 & 61 & 74 & 84 & 67 & 63 & 63 \\
Opt. 2-sided Robin & 264 & 25 & 24 & 25 & 23 & 24 & 23 \\
Opt. 2nd order & 19 & 18 & 17 & 18 & 17 & 17 & 18 \\
DN & $\mathrm{D}$ & 52 & 18 & 4 & 14 & 20 & 20 \\
ND & 46 & 34 & 22 & 4 & 30 & D & D \\
\hline
\end{tabular}

Finally, some results with more than 2 subdomains are presented. The computational domain is decomposed into vertical strips of the same width, with a diffusion coefficient that jumps at each interface: $v=0.001,1,0.001,1$, and so on. It is expected that the convergence detriorates as we increase the number of subdomains; the remedy would be using a coarse space correction [6]. In Table 4, we compare the different methods as iterative solvers, without any kind of coarse correction. The method labeled DN best refers to imposing the Dirichlet condition for each interface on the subdomain with the smaller diffusion coefficient (this guarantees convergence without relaxation when using two subdomains). On each interface, the optimal relaxation parameter from the analysis with two subdomains is employed. It can be noted from Table 4 that the convergence of this Dirichlet-Neumann method deteriorates as we increase the number of subdomains. The most robust method is again with the optimized second order transmission conditions, which converges significantly faster than Dirichlet-Neumann for many subdomains.

It was shown in this paper that with a discontinuous diffusion coefficient, the optimized two-sided Robin conditions produce a better asymptotic convergence factor than the optimized second order conditions, both when the grid size $h$ is small (Theorem 7 and 9) and when the jump in the coefficient $\mu$ is large (Theorem 10). On the other hand, the numerical results in this subsection, and the additional results in [5], demonstrate that the second order conditions are more robust compared to the two-sided Robin conditions when breaking the symmetry of the decomposition and when using many subdomains.

Table 4 Number of iterations to reach a tolerance of $10^{-6}$, for an increasing number of subdomains, when $\boldsymbol{\mu}=\mathbf{1 0 0}, N=240$

\begin{tabular}{|l|c|c|c|c|c|c|c|}
\hline Number of subdomains & 2 & 4 & 6 & 8 & 10 & 12 & 16 \\
\hline Opt. Robin v.1 & 72 & 74 & 86 & 114 & 141 & 170 & 230 \\
Opt. Robin v.2 & 15 & 23 & 29 & 35 & 41 & 47 & 58 \\
Opt. 2-sided Robin & 7 & 9 & 10 & 15 & 17 & 25 & 71 \\
Opt. 2nd order & 8 & 10 & 11 & 13 & 15 & 20 & 40 \\
DN best & 4 & 11 & 15 & 23 & 35 & 51 & $>1000$ \\
DN best no relaxation & 8 & 11 & 13 & 19 & 25 & 40 & $>1000$ \\
\hline
\end{tabular}




\section{Conclusion}

We derived and analyzed optimized Schwarz methods for a diffusion problem with discontinuous coefficient, using two non-overlapping subdomains. The transmission conditions are optimized to obtain fast convergence of the iteration, without changing the computational cost of each iteration. The solutions of the min-max problems are fully characterized and formulas for the best parameters are provided. When using an appropriate scaling, the performance of optimized Schwarz methods is shown to be very effective for strongly heterogeneous media. In particular, the optimization of two-sided transmission conditions seems to provide the best convergence when the coefficients are discontinuous; we exposed analytical and numerical evidence that the resulting convergence factor is mesh independent. We conjecture that these conclusions will hold as well in the more general case of advection-diffusion problems with discontinuous coefficients.

\section{References}

1. Bennequin, D., Gander, M.J., Halpern, L.: A homographic best approximation problem with application to optimized Schwarz waveform relaxation. Math. Comp. 78, 185-223 (2009)

2. Blayo, E., Halpern, L., Japhet, C.: Optimized Schwarz waveform relaxation algorithms with nonconforming time discretization for coupling convection-diffusion problems with discontinuous coefficients. In: Widlund, O.B., Keyes, D.E. (eds.) Domain Decomposition Methods in Science and Engineering XVI, Lecture Notes in Computational Science and Engineering, vol. 55, pp. 267-274. Springer (2006)

3. Calugaru, D.G., Tromeur-Dervout, D.: Non-overlapping DDMs to solve flow in heterogeneous porous media. In: Kornhuber, R., Hoppe, R.H.W., Péeriaux, J., Pironneau, O., Widlund, O.B., Xu, J. (eds.) Proceedings of the 15th International Domain Decomposition Conference, pp. 529-536. Springer, LNCSE (2003)

4. Dubois, O.: Optimized Schwarz methods with Robin conditions for the advection diffusion equation. In: Widlund, O.B., Keyes, D.E. (eds.) Domain Decomposition Methods in Science and Engineering XVI, Lecture Notes in Computational Science and Engineering, vol. 55, pp. 181-188. Springer (2006)

5. Dubois, O.: Optimized Schwarz methods for the advection-diffusion equation and for problems with discontinuous coefficients. Ph.D. thesis, McGill University (2007)

6. Dubois, O., Gander, M.J., Loisel, S., St-Cyr, A., Szyld, D.B.: The optimized Schwarz method with a coarse grid correction. SIAM J. Sci. Comp. 34(1), A421-A458 (2012)

7. Faille, I., Flauraud, E., Nataf, F., Schneider, F., Willen, F.: Optimized interface conditions for sedimentary basin modeling. In: N.D. et al. (eds.) Proceedings of the 13th International Conference on DDM, pp. 461-468 (2001)

8. Falco, C.D., O'Riordan, E.: Interior layers in a reactiondiffusion equation with a discontinuous diffusion coefficient. Int. J. Num. Anal. Mod. 7(3), 444-461 (2010)

9. Farhat, C., Roux, F.X.: A method of Finite Element Tearing and Interconnecting and its parallel solution algorithm. Int. J. Numer. Meth. Eng. 32, 1205-1227 (1991)

10. Gander, M.J.: Optimized Schwarz methods for Helmholtz problems. In: 13th International Conference on Domain Decomposition, pp. 245-252 (2001)

11. Gander, M.J.: Optimized Schwarz methods. SIAM J. Numer. Anal. 44(2), 699-731 (2006)

12. Gander, M.J., Halpern, L.: Absorbing boundary conditions for the wave equation and parallel computing. Math. Comp. 74(249), 153-176 (2004)

13. Gander, M.J., Halpern, L.: Optimized Schwarz waveform relaxation methods for advection reaction diffusion problems. SIAM J. Numer. Anal. 45(2), 666-697 (2007)

14. Gander, M.J., Halpern, L., Kern, M.: A Schwarz waveform relaxation method for advection-diffusionreaction problems with discontinuous coefficients and non-matching grids. In: Widlund, O.B., Keyes, 
D.E. (eds.) Domain Decomposition Methods in Science and Engineering XVI, Lecture Notes in Computational Science and Engineering, vol. 55. Springer (2006)

15. Gander, M.J., Halpern, L., Nataf, F.: Optimal Schwarz waveform relaxation for the one dimensional wave equation. SIAM J. Numer. Anal. 41(5), 1643-1681 (2003)

16. Gander, M.J., Magoulès, F., Nataf, F.: Optimized Schwarz methods without overlap for the Helmholtz equation. SIAM J. Sci. Comput. 24(1), 38-60 (2002)

17. Gerardo-Giorda, L., Nataf., F.: Optimized Schwarz methods for unsymmetric layered problems with strongly discontinuous and anisotropic coefficients. J. Numer. Math. 13(4), 265-294 (2005)

18. Gerardo-Giorda, L., Nataf, F.: Optimized algebraic interface conditions in domain decomposition methods for strongly heterogeneous unsymmetric problems. In: Widlund, O.B., Keyes, D.E. (eds.) Domain Decomposition Methods in Science and Engineering XVI, Lecture Notes in Computational Science and Engineering, vol. 55. Springer (2006)

19. Gerardo-Giorda, L., Tallec, P.L., Nataf, F.: A Robin-Robin preconditioner for strongly heterogeneous advection-diffusion problems. In: Herrera, I., Keyes, D.E., Widlund, O.B., Yates, R. (eds.) 14th International Conference on Domain Decomposition Methods, Cocoyoc, Mexico (2002)

20. Hagstrom, T., Tewarson, R.P., Jazcilevich, A.: : Numerical experiments on a domain decomposition algorithm for nonlinear elliptic boundary value problems. Appl. Math. Lett. 1(3) (1988)

21. Ibrahima, C.: Décomposition de domaines pour des structures hétérogénes. Ph.D. thesis, Université Paris-Nord - Paris XIII. Laurence Halpern (2009). http://tel.archives-ouvertes.fr/tel-00582621/fr/

22. Japhet, C.: Optimized Krylov-Ventcell method. Application to convection-diffusion problems. In: Bjørstad, P.E., Espedal, M.S., Keyes, D.E. (eds.) Proceedings of the 9th International Conference on Domain Decomposition Methods, pp. 382-389. ddm.org (1998)

23. Japhet, C., Nataf, F.: The best interface conditions for domain decomposition methods: Absorbing boundary conditions. In: Tourrette, L. (ed.) Artificial Boundary Conditions, with Applications to Computational Fluid Dynamics Problems Nova Science (2000)

24. Japhet, C., Nataf, F., Rogier, F.: : The optimized order 2 method. application to convection-diffusion problems. Future Generation Computer Systems FUTURE, vol. 18 (2001)

25. Japhet, C., Nataf, F., Roux, F.X.: The Optimized Order 2 Method with a coarse grid preconditioner. Application to convection-diffusion problems. In: Bjørstad, P., Espedal, M., Keyes, D. (eds.) 9th International Conference on Domain Decompositon Methods in Science and Engineering, pp. 382-389. Wiley (1998)

26. Lions, P.L.: On the Schwarz alternating method. III: A variant for nonoverlapping subdomains. In: Chan, T.F., Glowinski, R., Périaux, J., Widlund, O. (eds.) 3rd International Symposium on Domain Decomposition Methods for Partial Differential Equations, held in Houston, Texas, March 20-22, 1989. SIAM, Philadelphia (1990)

27. Maday, Y., Magoulès, F.: Non-overlapping additive Schwarz methods tuned to highly heterogeneous media. Comptes Rendus a l'Académie des Sciences 341(11), 701-705 (2005)

28. Maday, Y., Magoules, F.: Improved ad hoc interface conditions for Schwarz solution procedure tuned to highly heterogenous media. Appl. Math. Model. 30(8), 731-743 (2006)

29. Maday, Y., Magoules, F.: Optimized Schwarz methods without overlap for highly heterogeneous media. Comput. Methods Appl. Mech. Eng. 196(8), 1541-1553 (2007)

30. Marini, L.D., Quarteroni, A.: A relaxation procedure for domain decomposition methods using finite elements. Numer. Math. 5, 575-598 (1989)

31. Martin, V.: An optimized Schwarz waveform relaxation method for unsteady convection diffusion equation. Appl. Numer. Math. 52(4), 401-428 (2005)

32. Nataf, F., Rogier, F.: Factorization of the convection-diffusion operator and the Schwarz algorithm. $M^{3}$ AS 5(1), 67-93 (1995)

33. Smith, B.F., Bjorstad, P.E., Gropp, W.: Domain Decomposition: Parallel Multilevel Methods for Elliptic Partial Differential Equations. Cambridge University Press (1996)

34. Tang, W.P.: Generalized Schwarz splittings. SIAM J. Sci. Stat. Comp. 13(2), 573-595 (1992)

35. Toselli, A., Widlund, O.: Domain Decomposition Methods - Algorithms and Theory, Springer Series in Computational Mathematics, vol. 34. Springer (2004) 